\title{
BLACK HOLE-GALAXY CORRELATIONS WITHOUT SELF-REGULATION
}

\author{
Daniel Anglés-Alcázar ${ }^{1}$, Feryal ÖZel ${ }^{2,3}$, and Romeel Davé ${ }^{2,4,5,6}$ \\ ${ }^{1}$ Department of Physics, University of Arizona, Tucson, AZ 85721, USA; anglesd@email.arizona.edu \\ ${ }^{2}$ Astronomy Department, University of Arizona, Tucson, AZ 85721, USA \\ ${ }^{3}$ Radcliffe Institute for Advanced Study, Harvard University, Cambridge, MA 02138, USA \\ ${ }^{4}$ University of the Western Cape, Bellville, Cape Town 7535, South Africa \\ ${ }^{5}$ South African Astronomical Observatories, Observatory, Cape Town 7925, South Africa \\ ${ }^{6}$ African Institute for Mathematical Sciences, Muizenberg, Cape Town 7945, South Africa \\ Received 2012 November 30; accepted 2013 March 18; published 2013 May 17
}

\begin{abstract}
Recent models of black hole growth in a cosmological context have forwarded a paradigm in which the growth is self-regulated by feedback from the black hole itself. Here we use cosmological zoom simulations of galaxy formation down to $z=2$ to show that such strong self-regulation is required in the popular spherical Bondi accretion model, but that a plausible alternative model in which black hole growth is limited by galaxy-scale torques does not require self-regulation. Instead, this torque-limited accretion model yields black holes and galaxies evolving on average along the observed scaling relations by relying only on a fixed, $5 \%$ mass retention rate onto the black hole from the radius at which the accretion flow is fed. Feedback from the black hole may (and likely does) occur, but does not need to couple to galaxy-scale gas in order to regulate black hole growth. We show that this result is insensitive to variations in the initial black hole mass, stellar feedback, or other implementation details. The torque-limited model allows for high accretion rates at very early epochs (unlike the Bondi case), which if viable can help explain the rapid early growth of black holes, while by $z \sim 2$ it yields Eddington factors of $\sim 1 \%-10 \%$. This model also yields a less direct correspondence between major merger events and rapid phases of black hole growth. Instead, growth is more closely tied to cosmological disk feeding, which may help explain observational studies showing that, at least at $z \gtrsim 1$, active galaxies do not preferentially show merger signatures.
\end{abstract}

Key words: black hole physics - galaxies: active - galaxies: evolution - quasars: general

Online-only material: color figures

\section{INTRODUCTION}

There is by now ample evidence that supermassive black holes reside at the center of most sizeable galaxies (e.g., Ferrarese \& Ford 2005). During the past two decades, observations of local galaxies have established a number of well-defined relationships between the mass of the central black hole and stellar properties of the host galaxy such as the mass of the central bulge $\left(M_{\mathrm{BH}}-M_{\text {bulge }}\right.$ relation; Magorrian et al. 1998; Häring \& Rix 2004), the concentration or Sérsic index (Graham et al. 2001), the bulge velocity dispersion $\left(M_{\mathrm{BH}}-\sigma\right.$ relation; Ferrarese \& Merritt 2000; Tremaine et al. 2002; Gültekin et al. 2009; McConnell et al. 2011), the virial bulge mass (Marconi \& Hunt 2003), the spheroid binding energy (Aller \& Richstone 2007), and even the mass of the dark matter halo (Ferrarese 2002). The tightness of these correlations suggests that there is a close connection between the formation and evolution of galaxies and their central black holes. Moreover, the similarity between the global cosmic star formation history (Madau et al. 1996) and the evolution of quasar abundances (Boyle \& Terlevich 1998), as well as the apparent connection of starburst events and active galactic nuclei (AGNs) in individual objects (Kauffmann et al. 2003), provides further support for a link between galaxy formation and accretion onto a central black hole. Understanding this connection can have broad implications in galaxy evolution and the formation of massive black holes in the early universe.

Analytic arguments suggest that the observed black hole-galaxy correlations might arise from the coupling of energy and/or momentum from radiation emitted by the black hole accretion process to the surrounding galaxy (a process known as black hole or AGN feedback), leading to the idea of self-regulated growth and the co-evolution of black holes and galaxies (Silk \& Rees 1998; Fabian 1999; King 2003; Begelman \& Nath 2005; Murray et al. 2005; McQuillin \& McLaughlin 2012). The energy produced by accretion onto the black hole can easily exceed the binding energy of its parent galaxy; therefore, even if a small fraction of this energy is transferred to the surrounding gas, black hole feedback can have a profound effect on the evolution of its host galaxy (Fabian 2012). This interpretation has been particularly attractive for galaxy formation models since both semianalytic models and cosmological hydrodynamic simulations require the injection of additional energy to prevent the overcooling of gas and the subsequent runaway formation of stars (e.g., Croton et al. 2006; Somerville et al. 2008; Gabor et al. 2011; Teyssier et al. 2011). AGN feedback is often invoked as the source of this energy.

Motivated by these ideas, an increasing number of studies have now incorporated models for black hole growth and feedback into idealized galaxy scale simulations as well as full cosmological simulations (e.g., Springel et al. 2005; Di Matteo et al. 2008; Booth \& Schaye 2009; Debuhr et al. 2011; Kim et al. 2011; Power et al. 2011; Choi et al. 2012; Dubois et al. 2012). Since the numerical resolution required to self-consistently follow gas inflows from $\sim 10 \mathrm{kpc}$ hostgalaxy scales down to the central black hole $\left(\sim 10^{-6}\right.$ pc for the Schwarzschild radius of a $10^{7} M_{\odot}$ black hole) is out of reach for current computers, simulations must rely on "sub-grid" models to infer black hole accretion rates based on the physical properties of the surrounding gas at the scales resolved in the simulation. The majority of galaxy formation simulations to date have employed black hole accretion prescriptions based on the spherical Bondi-Hoyle-Lyttleton parameterization (hereafter "Bondi rate"; Hoyle \& Lyttleton 1939; Bondi \& Hoyle 1944; 
Bondi 1952), with the exception of some recent models that incorporate the physics of angular momentum transport in non-spherically symmetric flows (e.g., Debuhr et al. 2011; Power et al. 2011). A variety of feedback models have been implemented to date, including the injection of a fraction of the accretion luminosity as thermal energy into the gas surrounding the black hole (Springel et al. 2005; Sijacki et al. 2007; Johansson et al. 2009), the injection of momentum from optically thick radiation fields (Debuhr et al. 2011), heating and radiation pressure from X-ray generated by the AGN (Hambrick et al. 2011; Kim et al. 2011; Choi et al. 2012), and the injection of mass and/or momentum from AGN-driven winds or radio jets (Choi et al. 2012; Debuhr et al. 2012; Dubois et al. 2012).

The success of coupled black hole accretion and feedback models in accounting for a number of observations, including black hole mass functions, Eddington ratio distributions, and black hole-galaxy correlations (e.g., Di Matteo et al. 2005; Hopkins et al. 2006), has contributed to establishing a paradigm in which black hole growth is self-regulated by feedback from the black hole itself. There is, however, no direct observational evidence to date for such self-regulated growth of black holes due to their own feedback (Alexander \& Hickox 2012). AGN feedback acting in the kinetic or radio mode is commonly observed in the form of radio-emitting relativistic jets, and its effects on the hot gas in galaxies and clusters are relatively well understood. The radiative or quasar feedback mode has been identified by the presence of highly blueshifted absorption and emission lines and broad line wings, but the overall impact of quasar-driven winds on the host galaxy remains uncertain (Fabian 2012). Even if AGN feedback is effective in quenching star formation in galaxies and suppressing cooling flows in groups and clusters (as required by galaxy formation models), there is a priori no reason for AGN feedback to be the dominant physical process regulating black hole growth in galaxies.

In the presence of a non-zero net angular momentum of the surrounding gas, black hole feeding likely occurs through viscous transport in a Keplerian accretion disk (e.g., Shakura \& Sunyaev 1973; Balbus \& Hawley 1998). Therefore, it is plausible that most of the feedback energy and momentum escape along the polar direction without significantly affecting further accretion (e.g., Ohsuga et al. 2005). In order to allow for significant black hole growth, the accretion disk must be continuously replenished by gas inflows from larger scales. The angular momentum of the gas and competition with star formation are known to represent significant barriers to the transport of gas from galactic scales down to the black hole accretion disk (e.g., Thompson et al. 2005; Jogee 2006; Hopkins $\&$ Quataert 2010). Thus, the rate of angular momentum transport relative to the local star formation rate (SFR) may well be the dominant physical process governing black hole growth in galaxies, even if AGN feedback is acting at some level (e.g., Escala 2006, 2007; Power et al. 2011).

Numerical simulations have shown that large-scale tidal torques produced by galaxy interactions, galaxy mergers, and gravitational instabilities in self-gravitating disks can lead to efficient angular momentum transport and the rapid inflow of gas into the central kiloparsec of galaxies (e.g., Hernquist 1989; Shlosman et al. 1989; Barnes \& Hernquist 1992). At smaller scales, large-scale torques become less efficient and additional mechanisms are required. Using multiple nested galaxy scale simulations of progressively higher resolution, Hopkins \& Quataert (2010) showed that a series of gravitational instabilities can generate net inflow rates of up to $1-10 M_{\odot} \mathrm{yr}^{-1}$ down to sub-parsec scales $(\lesssim 0.1 \mathrm{pc})$. They found that nonaxisymmetric perturbations to the stellar gravitational potential produce orbit crossings and shocks in the gas that can be efficient in removing angular momentum even at scales $\lesssim 10 \mathrm{pc}$. Hopkins \& Quataert (2011) derived analytic expressions for the loss of angular momentum in the presence of such shocks and presented an analytical "gravitational torque" model for the resulting gas inflow rates. This analytic model was found to reproduce gas inflow rates at $\lesssim 0.1$ pc scales from simulations with significantly less scatter than the Bondi parameterization for the total enclosed mass within a given radius.

The goal of this paper is to investigate the growth of supermassive black holes due to gravitational-torque-driven accretion in a cosmological context and determine whether this growth mechanism can account for the observed black hole-galaxy correlations without the requirements of AGN feedback and selfregulated growth. Our approach consists of combining highresolution cosmological hydrodynamic simulations together with analytic models of black hole accretion in post-processing. This simplification allows us to follow the growth of galaxies and black holes from early epochs down to $z=2$ without making any prior assumptions about the effects of AGN feedback on galactic scales. By comparing predictions from the Bondi and gravitational torque models, we evaluate the relative importance of feedback and angular momentum transport in regulating cosmological black hole growth. In addition, we discuss the implications of different black hole accretion models on the link between major galaxy mergers and rapid phases of black hole growth, as well as the growth of black hole seeds in the early universe. The observed black hole-galaxy correlations are regarded here as a strong constraint for theories of black hole growth and galaxy evolution but not as a mandatory outcome of AGN feedback, as it is commonly assumed. Given that both star formation and black hole accretion histories peak at $z \sim 2$, our simulations target the critical epoch when such correlations were likely established.

This paper is organized as follows. We describe our simulations and main analysis procedures in Section 2. In Section 3, we present an overview of our simulated galaxies and describe their main physical properties. In Section 4, we evaluate the Bondi and gravitational torque models and show how the inferred black hole accretion rates relate to the physical properties and evolution of the host galaxies. In Section 5, we use the observed black hole-galaxy correlations to put constraints on black hole accretion in cosmological timescales and evaluate the effects of AGN feedback as required by different black hole models. In Sections 6 and 7, we show that the gravitational torque model naturally yields the observed black hole-galaxy correlations regardless of the initial masses of black holes and galaxies, and without the need for self-regulation by galaxy-scale AGN feedback. Finally, we summarize our results in Section 8 .

\section{SIMULATIONS}

We use the extended version of the GADGET-2 code (Springel 2005) that has been fully described in Oppenheimer \& Davé (2008). GADGET-2 combines a TreePM algorithm for computing gravitational forces with an entropy-conserving formulation of smoothed particle hydrodynamics (SPH; Springel \& Hernquist 2002). We include radiative cooling from primordial gas assuming ionization equilibrium as in Katz et al. (1996) and metal-line cooling based on Sutherland \& Dopita (1993). Photoionization heating is modeled via a spatially uniform, 
optically thin UV background (Haardt \& Madau 2001) starting at $z=9$.

Star formation and feedback are modeled in GADGET-2 through a sub-grid prescription. Gas particles that are sufficiently dense to become Jeans unstable are treated as a multi-phase fluid with cold, dense clumps embedded within a hot phase medium (McKee \& Ostriker 1977; Springel \& Hernquist 2003). Stars form from the cold phase, and feedback from Type II supernovae causes evaporation of cold clumps into the hot phase (which can condense back onto cold clumps) and metal enrichment. The resulting SFRs are in accord with the observed Kennicutt (1998) relation. We also account for energy and metal feedback from Type Ia supernovae and mass loss and metal enrichment from asymptotic giant branch stars, tracking the production of four metal species $(\mathrm{C}, \mathrm{O}, \mathrm{Si}$, and $\mathrm{Fe}$ ) separately as described in Oppenheimer \& Davé (2008). In addition, our simulations include a galactic outflow mechanism that imparts kinetic energy to gas particles. The outflow rates scale with galactic velocity dispersion, $\sigma$, and the ratio of material entering the wind versus forming stars (i.e., the mass loading factor) scales as $1 / \sigma$, as in the momentum-driven wind case (Murray et al. 2005). An on-the-fly galaxy finder is used to compute galaxy masses, which are converted to $\sigma$ using standard relations (Mo et al. 1998).

Overall, this treatment of metal production and galactic winds has been successful in reproducing a broad range of observations including the chemical enrichment of the intergalactic medium at $z>2$ (Oppenheimer \& Davé 2006, 2008), the luminosity function of high-redshift galaxies (Davé et al. 2006; Finlator et al. 2007), and the galaxy mass-metallicity relation (Finlator \& Davé 2008; Davé et al. 2011). In addition, the high-resolution zoom simulations presented here have been successful in reproducing many of the star formation, morphological, and kinematic properties of $z=2$ galaxies (Anglés-Alcázar et al. 2013).

We note that our simulations do not include prescriptions for black hole growth and the effects of AGN feedback. Our goal is to use galaxy formation simulations together with analytic black hole accretion models to study the physical processes governing cosmological black hole growth, based only on the observed black hole-galaxy correlations, and with no implicit assumptions about the amount and efficiency of feedback from the accretion process.

\subsection{Simulation Runs}

In this work we use the "zoom-in" technique (e.g., Navarro $\&$ White 1994) to run cosmological simulations and resolve the physical conditions at the inner kiloparsec of galaxies over cosmic time. We first ran an intermediate-resolution cosmological simulation with $2 \times 256^{3}$ gas +dark matter particles in a $\left[24 h^{-1} \mathrm{Mpc}\right]^{3}$ comoving box. This simulation allows us to characterize the population from which galaxies are selected for resimulation. We chose two galaxies at $z=2$ characterized by similar masses but different morphologies, environments, and merger histories. We identified all particles within the virial radius of each galaxy and traced them back to their locations on the initial grid, where they mark the refinement region. Zoom initial conditions were then generated by populating the refinement region with a larger number of lower mass particles (each particle is replaced by $8^{l}$, where $l$ is the zoom level), and adding the additional small-scale fluctuations appropriate to the new Nyquist frequency. In order to reduce numerical artifacts due to the difference in particle masses and to make sure that the large-scale gravitational torques acting on the target halos are accurately represented, the high-resolution region was significantly enlarged by an iterative "cleaning" procedure and surrounded by two nested, concentric layers of progressively lower resolution. After running the simulations, we enlarged our galaxy sample by including six additional galaxies that were found well within the high-resolution regions, confirming that there is no contamination of low-resolution particles within the virial radius of each galaxy.

Our zoom simulations have an effective resolution equivalent to $2 \times 1024^{3}$ particles homogeneously distributed in a $\left[24 h^{-1} \mathrm{Mpc}\right]^{3}$ box, with (high-resolution) gas particle mass $m_{\text {gas }} \approx 2.3 \times 10^{5} M_{\odot}$, dark matter particle mass $m_{\mathrm{DM}} \approx$ $1.2 \times 10^{6} M_{\odot}$, and softening length $\epsilon \approx 0.47 \mathrm{~h}^{-1} \mathrm{kpc}$ comoving (or $\sim 224 \mathrm{pc}$ physical at $z=2$ ). All results presented in this paper (except where indicated) correspond to simulations including our preferred model for galactic outflows, based on momentum-conserving scalings as explained above. In order to identify the possible effects of winds in our results, we also ran all simulations without winds. Additionally, we ran simulations with $2 \times 512^{3}$ effective resolution (i.e., two times lower spatial resolution and eight times lower mass resolution) for all galaxies to test for numerical convergence.

Throughout this paper we assume a $\Lambda \mathrm{CDM}$ cosmology with parameters $\Omega_{\Lambda}=0.72, \Omega_{\mathrm{M}}=0.28, \Omega_{\mathrm{b}}=0.046$, $h=0.7, \sigma_{8}=0.82$, and $n=0.96$, consistent with the five-year Wilkinson Microwave Anisotropy Probe concordance cosmology (Komatsu et al. 2009).

\subsection{Analysis of Simulations}

We produced over 230 snapshot files at time intervals ranging from $\sim 5$ to $25 \mathrm{Myr}$ during the simulation. We identify galaxies by means of the Spline Kernel Interpolative Denmax algorithm $\left(\operatorname{SKID}^{7}\right)$ at each redshift snapshot independently (see Keres et al. 2005 for a description). Our simulated galaxies are thus defined as bound groups of star-forming gas particles (i.e., gas particles with densities above the threshold for star formation) and star particles. We initially associate each SKID-identified galaxy with a dark matter halo by using a spherical overdensity algorithm, defining the virial radius as the radius enclosing a mean density given by Kitayama \& Suto (1996). Overlapping halos are then grouped together so that, by construction, every halo in our final catalog has a central galaxy (the most massive galaxy) and a number of satellite galaxies. Beginning at $z=2$, we reconstruct the evolution of each individual galaxy back in time by identifying each galaxy's most massive progenitor at all redshifts. All galaxies presented here are centrals.

When calculating morphological and kinematic properties of each galaxy as a function of time, we take the position of the most bound gas particle as the center of the galaxy, which corresponds to the position of the black hole. This results in a physically more meaningful and a significantly more stable definition compared to the galaxy center computed by SKID, especially during close galaxy encounters and galaxy mergers. Since we are interested in the evolution of galaxies and black holes over cosmological timescales, we smooth the time evolution of all physical quantities and extract their main evolutionary features by calculating averages over time intervals of $50 \mathrm{Myr}$ (except otherwise noted). Finally, we impose a resolution limit on our sample of galaxies by requiring that they contain at least 100 gas particles and 100 star particles within the inner kiloparsec. In this way, we ensure that the morphology and the physical conditions

\footnotetext{
http://www-hpcc.astro.washington.edu/tools/skid.html
} 

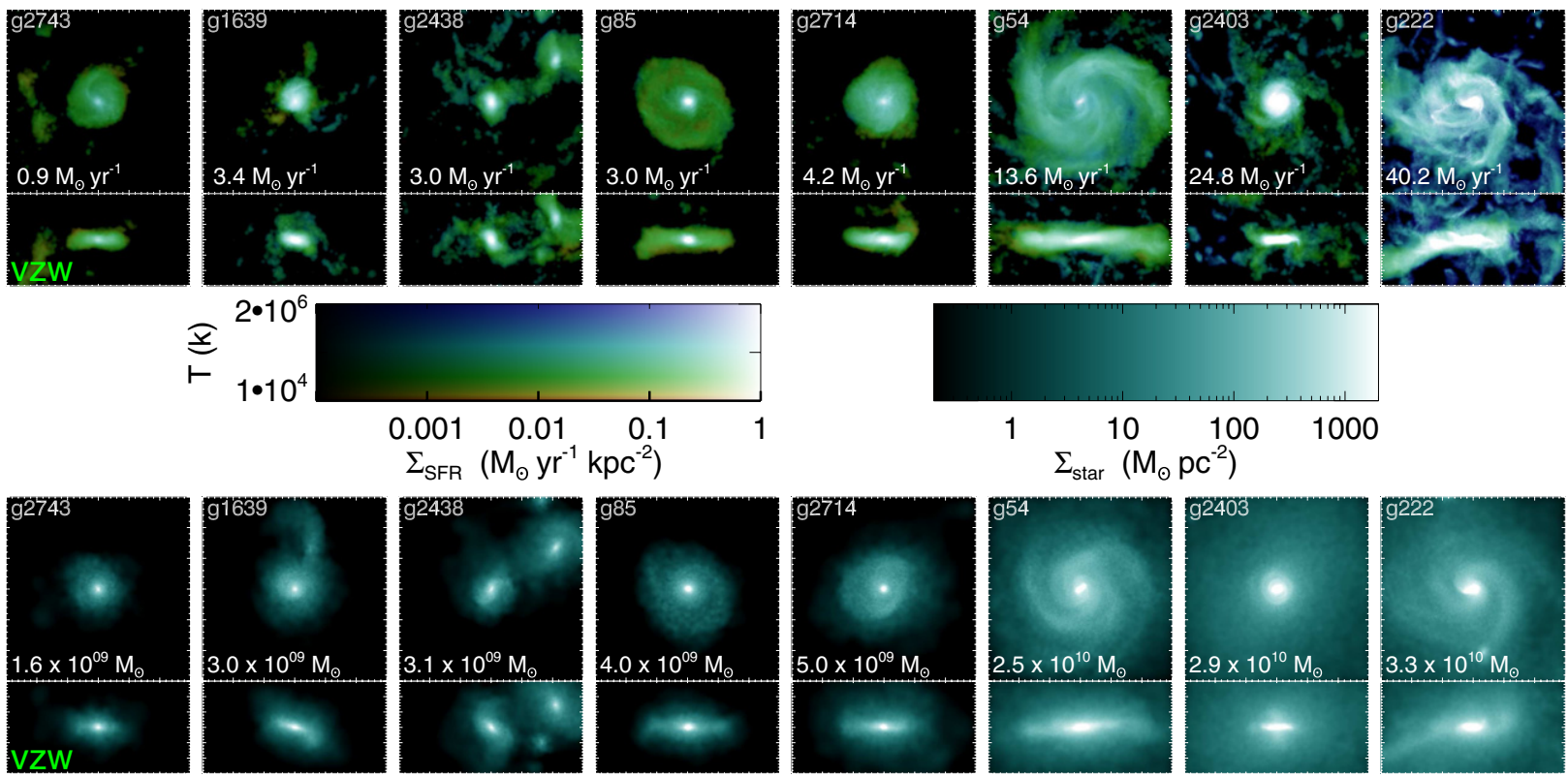

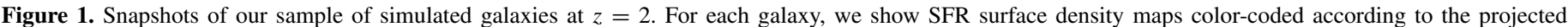

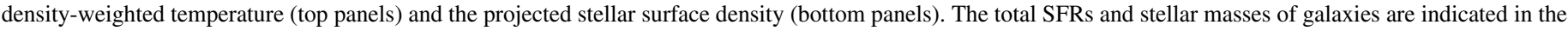

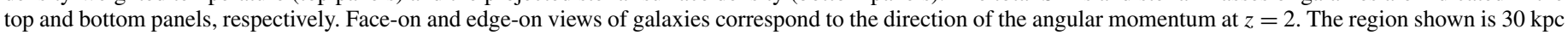
(physical) across.

(A color version of this figure is available in the online journal.)

at the centers of galaxies can be accurately characterized for the purposes of this work. Therefore, lower mass galaxies take part of the analyses and results only until they are resolved at later times.

\section{GALAXY PROPERTIES}

Our zoom simulations reproduce many characteristic features of observed $z \approx 2$ star-forming galaxies. The properties of these galaxies have been analyzed in detail in a separate paper (Anglés-Alcázar et al. 2013), where we show that strong stellar winds are required in order to maintain high gas fractions, redistribute star-forming gas over larger scales, and increase the velocity dispersion of simulated galaxies, in better agreement with the large, extended, rotation-dominated yet turbulent starforming disks of the SINS survey (Förster Schreiber et al. 2009).

Here, we provide a general description of all simulated galaxies and then focus on the physical conditions at their centers, since they eventually determine the accretion rates onto the central black hole.

\subsection{Global Properties}

Figure 1 gives a visual impression of our galaxy sample by showing the distribution of the star-forming gas and stellar contents at $z=2$. For most galaxies, the star-forming gas lies primarily in a thick, rotationally supported disk, with sizes ranging from $\sim 5$ to $\sim 25 \mathrm{kpc}$. A wide range of morphologies can be identified, including prominent spiral arms (galaxy g54), a very compact gas distribution (galaxy g2403), a pair of interacting galaxies (g2438), and a turbulent, highly disturbed galaxy (g222). The projected stellar distributions also reveal a wide variety of galaxy sizes and morphologies. Most galaxies appear to have a large-scale stellar disk surrounded by a significant spherical component.

At $z=2$, our simulated galaxies span about an order of magnitude in both stellar mass (from $\sim 1.6 \times 10^{9}$ to $\sim 3.3 \times$
$10^{10} M_{\odot}$ ) and SFR (from $\sim 1$ to $40 M_{\odot} \mathrm{yr}^{-1}$ ). Figure 2 shows the evolution of individual galaxies from $z=8$ to 2 in terms of their stellar mass, gas fraction, and specific SFR. Major mergers (identified as abrupt changes in the stellar mass of galaxies) can have a significant impact on the dynamical properties of galaxies but represent only a small fraction of the total mass growth down to $z=2$. Specific SFRs follow a common trend for all galaxies, decreasing from $\sim 10 \mathrm{Gyr}^{-1}$ at $z=8$ down to $\sim 1 \mathrm{Gyr}^{-1}$ at $z=2$, but their individual star formation histories are clearly different. Gas fractions evolve relatively slowly from nearly unity at $z=$ 8 down to $\sim 0.5$ at $z=2$, due to the early suppression of star formation by momentum-driven winds and the recycling of gas at later times. Overall, our simulated galaxies are characterized by a wide range of masses, morphologies, and star formation histories and are representative of "normal" $z=2$ systems (Anglés-Alcázar et al. 2013).

\subsection{The Central Kiloparsec}

In order to investigate the dominant processes governing black hole growth, it is crucial to understand the physical conditions at the inner regions of galaxies, how they evolve in time, and what the main evolutionary triggers are. Here, we evaluate the masses and morphologies of the stellar and gas contents of galaxies, as well as the specific SFRs within a radial distance $R_{0}=1 \mathrm{kpc}$ from their centers. Largescale gravitational torques produced by galaxy mergers and interactions are often invoked as triggers of star formation and AGN activity (e.g., Springel et al. 2005). The identification of galaxy mergers in cosmological simulations is, however, a nontrivial task in which the simple working definition of "galaxy" can result in a rather different timing of merger events or the temporal identification of close galaxy encounters as a merging system (Gabor et al. 2011). For the purposes of this work, we simply associate changes in galaxy properties with galaxy interactions and/or mergers by visual identification from gas 


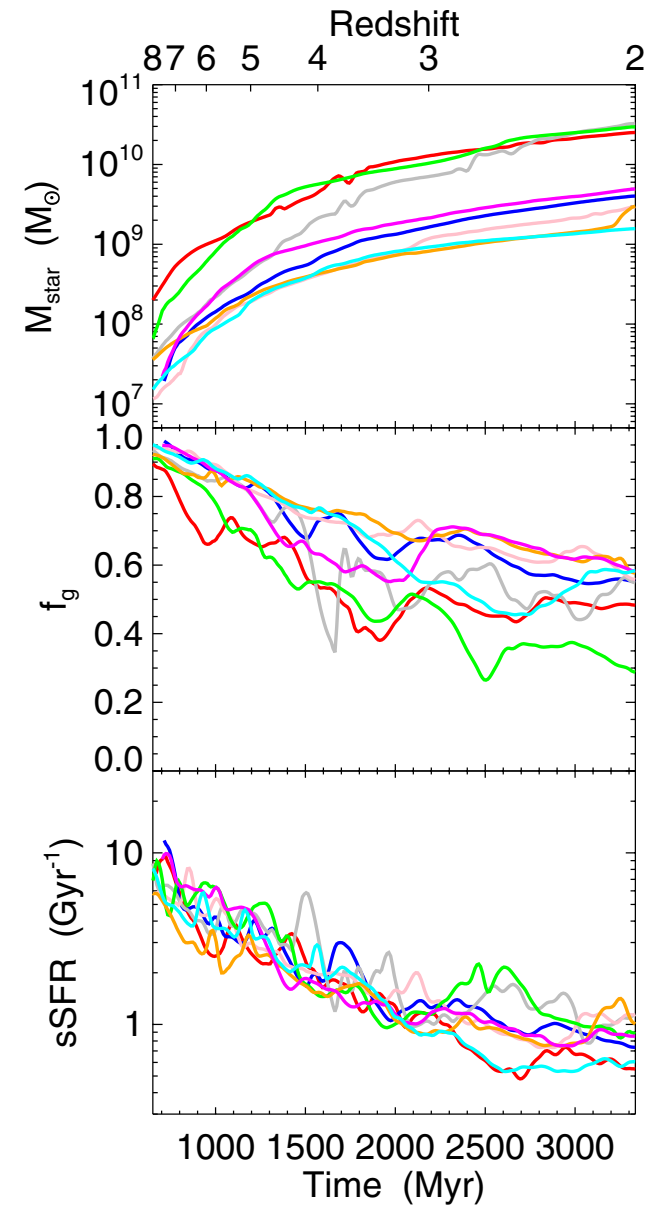

Figure 2. From top to bottom: stellar mass, gas fraction, and specific SFR as a function of time from $z=8$ to $z=2$ for all simulated galaxies. Each color corresponds to a different galaxy.

(A color version of this figure is available in the online journal.)

and stellar surface density plots (similar to those in Figure 1) at the appropriate snapshot times.

Figure 3 characterizes the masses and morphologies of the stellar and gas contents of galaxies g54 and g222 within the inner kiloparsec as a function of time. In the remainder of this section, we use these two galaxies to illustrate the analysis, but we note that our conclusions apply to all simulated galaxies. Galaxy g54 undergoes a relatively quiescent evolution below $z \approx 3$, after a merger that results in a significant increase in the stellar mass within $R_{0}$ ( $\sim 2000$ Myr after the big bang). Galaxy g222 is located in a denser environment and undergoes a more violent evolution, with mergers that result in the rapid increase of stellar and gas masses within $R_{0}$ at times $\sim 1500,1900,2700$, and 3000 Myr.

Here we parameterize the gas and stellar morphologies within the inner kiloparsec of galaxies based on the amount of rotational support, $\kappa_{\text {rot }}$, and the bulge mass fraction, $f_{\text {bulge }}$. We calculate $\kappa_{\text {rot }}$ as the fraction of kinetic energy in ordered rotation with respect to the total angular momentum within $R_{0}$ (e.g., Sales et al. 2010). Additionally, we estimate $f_{\text {bulge }}$ from a bulge-disk decomposition based on the full three-dimensional kinematic information of particles of either the gas or the stellar components. We calculate the azimuthal velocity $\left(v_{\phi}\right)$ of each particle with respect to the direction of the total angular momentum within $R_{0}$. In the presence of a spherical component supported by random motions, $v_{\phi}$ is expected to have a symmetric distribution with
$50 \%$ of the particles having velocities $v_{\phi}<0$ on average. Therefore, by adding up the masses of particles with $v_{\phi}<0$ (and multiplying by 2) we can get an estimate of the total mass in a spherical component. This will certainly underestimate $f_{\text {bulge }}$ in the case of rotating bulges, but it is a reasonable approximation for the purposes of this work.

Figure 3 shows that the gas component is more rotationally supported than the stellar component at all times, since the infalling gas can cool down and form a disk even if only a fraction of the initial angular momentum is retained. In the absence of strong perturbations, the stellar $\kappa_{\text {rot }}$ tends to increase as the stars form from the gas disk (e.g., galaxy g54 from $z=6 \rightarrow 3$ ). Galaxy interactions and mergers result in gas inflows toward the center of galaxies and leave their imprint in $\kappa_{\text {rot }}$ : an increase in the total gas mass within $R_{0}$ usually correlates with a decrease in $\kappa_{\text {rot }}$ (e.g., galaxy g222 at times $\sim 1500$ and $1900 \mathrm{Myr})$. As expected, $f_{\text {bulge }}$ anticorrelates with $\kappa_{\text {rot }}$, since both quantities are calculated based on particle kinematics. We see that galaxy interactions result in a temporary increase of the total gas mass, as well as the mass fraction in a spherical component. Correspondingly, the evolution of the specific SFR within the inner kiloparsec appears to be modulated by galaxy interactions and mergers. These events generally trigger temporary increases in the specific SFR that correlate with changes in the gas morphology and kinematics. The morphology of the stellar component can be affected by this enhanced star formation activity, since stars forming from low angular momentum gas after a merger can result in the increase of the stellar bulge component (e.g., galaxy g222 at $t \sim 1500 \mathrm{Myr}$ ).

In summary, global properties of simulated galaxies and their evolution on cosmological timescales are dominated by smooth accretion and wind-regulated star formation (Anglés-Alcázar et al. 2013). Despite this, galaxy interactions and mergers can have a significant impact on the morphology and star formation properties at the inner regions of galaxies. As we show in the next section, these events will have an effect on the inferred central black hole accretion rates and the evolution of AGN activity.

\section{CENTRAL ACCRETION RATES}

We infer the accretion rates onto black holes located at the center of each simulated galaxy based on two different accretion prescriptions. The first is the spherical Bondi accretion, in which the mass accretion rate is determined by the amount of matter captured gravitationally by the black hole. The second is the accretion rate driven by torques produced by gravitational instabilities at different scales in the inner galaxy. We utilize the analytic model developed by Hopkins \& Quataert (2011) to calculate the latter accretion rate. Since the evolution of the central kiloparsec of galaxies has been fully characterized, we can evaluate black hole accretion rates resulting from different physical processes and investigate the connection of AGN activity and host galaxy properties. As we show below, the use of a particular black hole accretion model in galaxy formation simulations can lead to rather different predictions on the galaxy-AGN connection.

We choose the most bound particle in each galaxy to represent the central black hole. However, in this set of simulations, we do not self-consistently update the black hole mass; therefore, we neglect the gravitational influence of the black hole at the scales resolved in the simulation. The Bondi radius (Bondi 1952) of a black hole of mass $M_{\mathrm{BH}}=10^{7} M_{\odot}$, assuming a sound speed $c_{\mathrm{s}}=30 \mathrm{~km} \mathrm{~s}^{-1}$, is $r_{\mathrm{B}}=G M_{\mathrm{BH}} / c_{\mathrm{s}}^{2} \approx 50 \mathrm{pc}$, which is below 

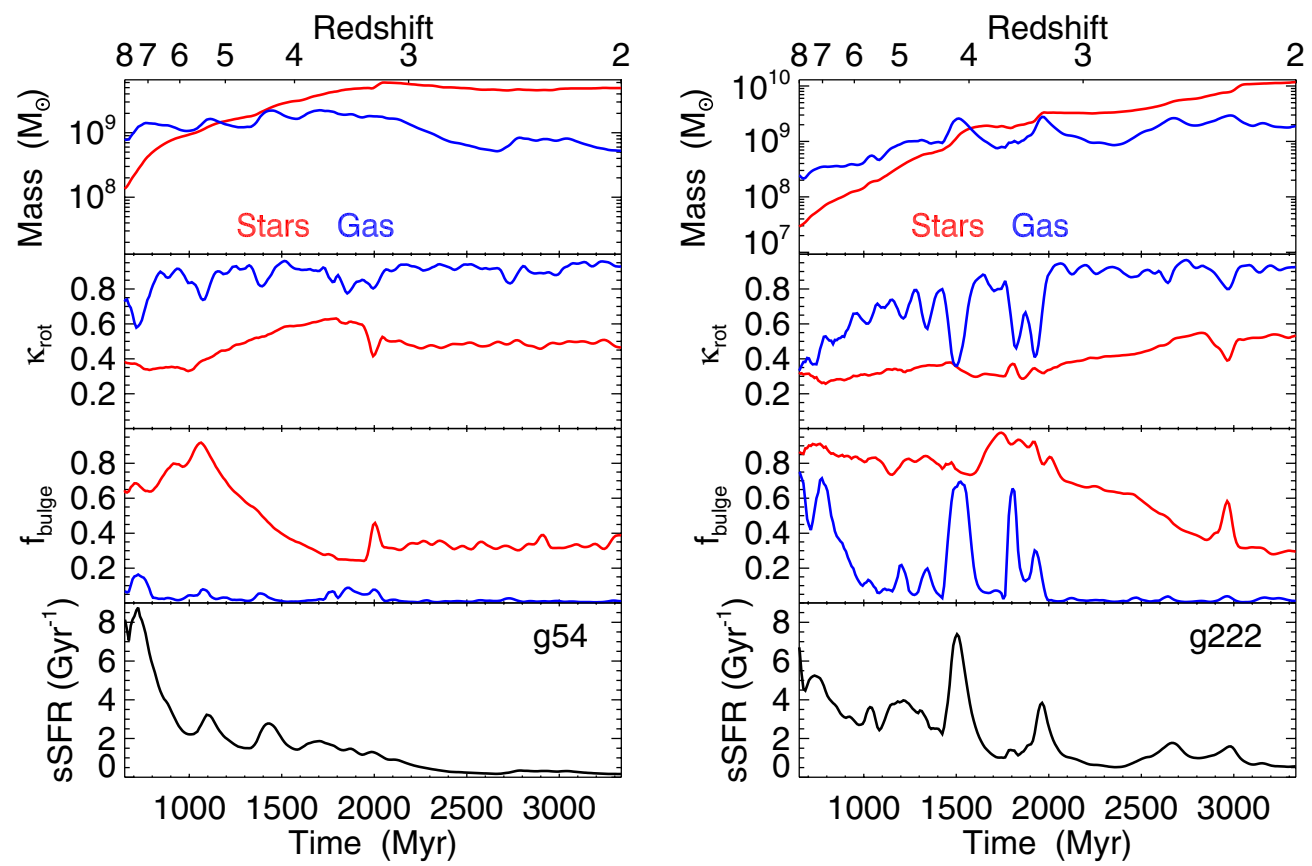

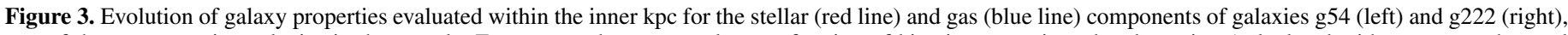

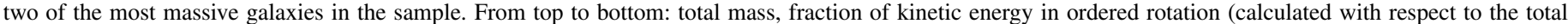
angular momentum of the galaxy), bulge mass fraction (mass fraction in a spherical component calculated from kinematic decomposition), and specific SFR.

(A color version of this figure is available in the online journal.)

the physical softening length in our simulations for the redshift range of interest $(\epsilon \approx 75-224 \mathrm{pc}$ at $z=8-2)$. Furthermore, the typical masses of central black holes that we infer for our simulated galaxies (see Section 5) are, at most, comparable to the mass of a few tens of gas particles, and we typically resolve the inner kiloparsec of galaxies with thousands of gas particles. Therefore, given the mass and force resolution in our simulations, we do not expect the lack of gravitational force from the central black hole to have a significant effect on accretion rate estimates. It can be argued, however, that a more critical issue is the lack of black hole feedback in our simulations. We will address the issue of feedback in Section 5.

\subsection{Bondi-Hoyle-Lyttleton Parameterization}

The Bondi model (Hoyle \& Lyttleton 1939; Bondi \& Hoyle 1944; Bondi 1952) is the most widely used prescription for black hole growth in galaxy formation simulations (e.g., Springel et al. 2005; Di Matteo et al. 2008). For a black hole of mass $M_{\mathrm{BH}}$, moving at velocity $v$ relative to a uniform distribution of gas with density $\rho$ and sound speed $c_{\mathrm{s}}$, the Bondi rate is given by

$$
\dot{M}_{\text {Bondi }}=\alpha \frac{4 \pi G^{2} M_{\mathrm{BH}}^{2} \rho}{\left(c_{\mathrm{s}}^{2}+v^{2}\right)^{3 / 2}},
$$

where $\alpha$ is a dimensionless parameter that is usually added to boost accretion rates and partially compensate for the relatively high mean gas temperatures resulting from the multi-phase subgrid model of star formation and/or the lack of the spatial resolution required to resolve the Bondi radius (Booth \& Schaye 2009; Johansson et al. 2009). The choice of this parameter, together with the initial black hole mass, can have a significant effect on the early growth of black holes. Here we use a constant value $\alpha=100$, similar to many previous studies (e.g., Springel et al. 2005). For comparison, we also explore the functional form introduced by Booth \& Schaye (2009), where $\alpha \propto \rho^{2}$ for gas at densities above the threshold for star formation ( $n>0.13 \mathrm{~cm}^{-3}$ in our simulations) and $\alpha=1$ for lower density, single-phase gas. We note that further modifications to the Bondi parameterization have been proposed for the case of efficient cooling and significant contribution of the surrounding halo to the total gravitational potential (Hobbs et al. 2012). Making the reasonable assumption that the black hole is located at the center of the potential well, we can get an estimate of the Bondi rate based on the properties (gas density and sound speed) of the most bound gas particle at the center of each simulated galaxy.

Figure 4 shows the evolution of the density and sound speed at the location of the black hole in galaxies g54 and g222. Beginning at $z=8$, the central density of galaxy g54 increases by over two orders of magnitude up to $\sim 250 \mathrm{~cm}^{-3}$ at the end of the last merger $(t \sim 2000 \mathrm{Myr})$ and then decreases rapidly down to $\sim 30 \mathrm{~cm}^{-3}$ at $z=2$. Prior to the last merger, the accretion of low-mass gas-rich satellites results in temporary increases of the central density that correlate with morphological changes and increases in specific SFRs as seen in Figure 3. The effects of mergers in the central density of galaxy g222 are more evident, with significant density peaks (up to $\sim 400 \mathrm{~cm}^{-3}$ ) clearly correlating with changes in the specific SFR within the inner kiloparsec.

We evaluate the relative velocity of the surrounding gas $(v)$ as an SPH-kernel weighted averaged with respect to the most bound gas particle, resulting in significantly lower values compared to the typical sound speed (Figure 4). For both galaxies, the evolution of the central sound speed resembles that of the density but with less than a factor of three variation during the simulation. We note that the large values of sound speed shown here are due to the effective equation of state resulting from the sub-grid prescription of star formation. This leads to a significant suppression of Bondi rates that is partially compensated by the addition of the boost factor $\alpha$ in Equation (1) (see Pelupessy et al. 2007 for an alternative approach). 

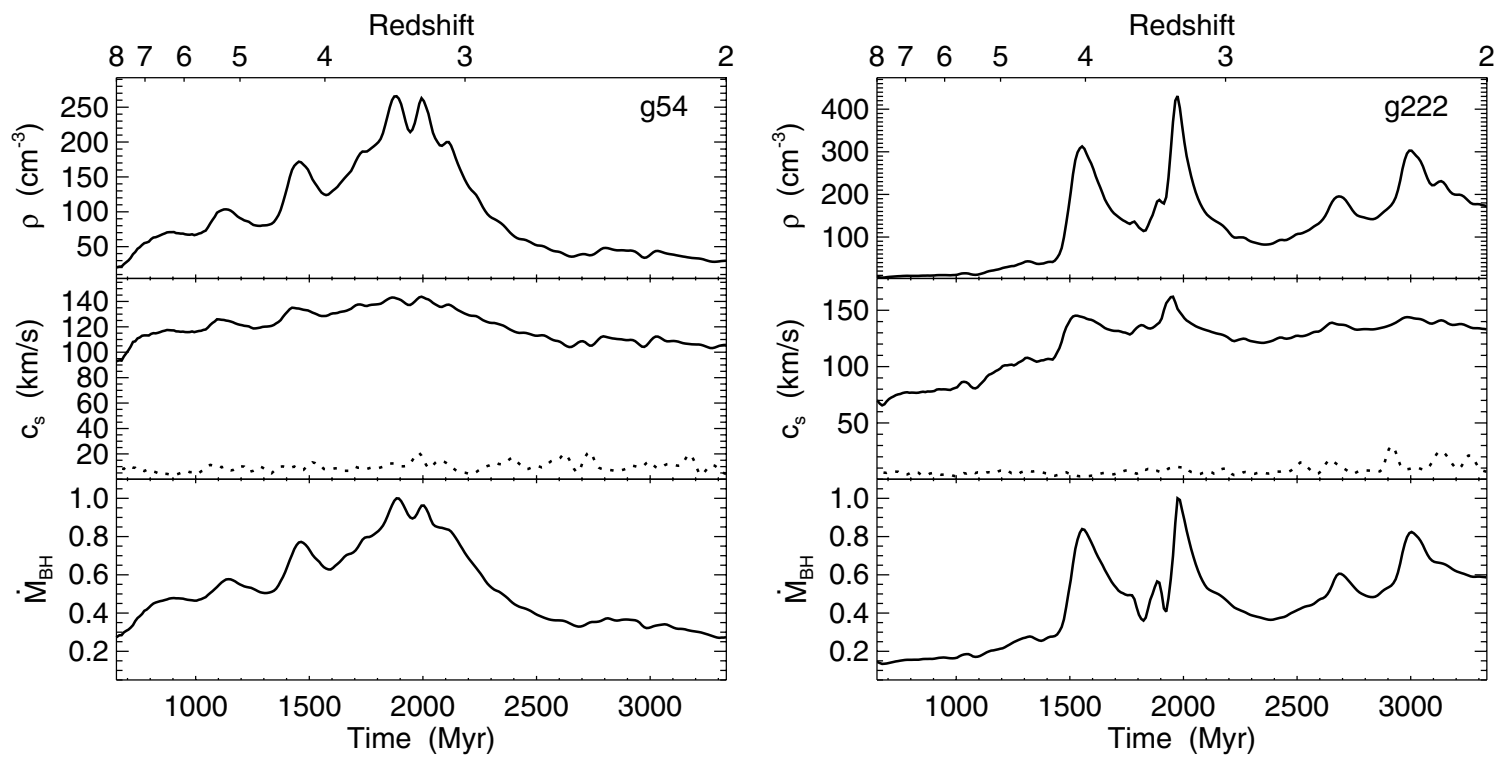

Figure 4. Inferred Bondi rates for galaxies g54 (left) and g222 (right). Top: gas density at the location of the most bound gas particle. Middle: sound speed at the location of the most bound gas particle (solid line) and relative velocity with respect to the surrounding gas (dotted line). Bottom: inferred black hole accretion rates using the Bondi-Hoyle-Lyttleton parameterization (Equation (1) with $\alpha=100$ ) and based on the gas properties at the location of the most bound gas particle, where we have used a constant black hole mass $M_{\mathrm{BH}}=10^{5} M_{\odot}$ at all times and normalized to the peak accretion rate.

The Bondi rates inferred for black holes located at the centers of galaxies g54 and g222 are shown in Figure 4. Since the goal here is to identify the physical drivers of black hole accretion, we simply evaluate Equation (1) for a nominal, constant black hole mass $M_{\mathrm{BH}}=10^{5} M_{\odot}$ at all times and show normalized Bondi rates relative to the peak value for each galaxy. This simplification allows us to avoid making assumptions about the actual growth of black holes at this stage and to focus only on how the evolution of the host galaxy relates to relative changes in black hole accretion according to the Bondi model. Figure 4 shows that the evolution of the normalized Bondi rates resembles that of the central density, and it is therefore imprinted with the effects of galaxy mergers. Since mergers invariably cause an increase in the central density of galaxies, the Bondi model predicts a direct connection between AGN activity and galaxy mergers, as has been found in many previous simulations (e.g., Di Matteo et al. 2005; Springel et al. 2005). Moreover, since star formation is also proportional to the gas density (the subgrid model is tuned to match the observed relation of Kennicutt 1998), the Bondi model provides support for a merger-driven scenario for the origin of starbursts and quasar phases (Hopkins et al. 2006).

\subsection{Accretion due to Gravitational Torques}

In any realistic flow with a non-zero net angular momentum, the gas is expected to settle down onto a rotationally supported disk. The rate at which the gas is accreted depends on the rate at which its angular momentum is removed. Here, we evaluate black hole accretion rates based on the analytic model of Hopkins \& Quataert (2011) that accounts for the angular momentum transport in the galactic disk.

The gravitational torque model predicts inflow rates at subparsec scales as a function of physical quantities evaluated at scales that can be resolved in the simulation. Based on this prescription, the black hole accretion rate (1) increases linearly with the total (stellar and gas) disk mass, $M_{\text {disk }}$, (2) depends strongly on the total (stellar and gas) disk mass fraction, $f_{\text {disk }}$, (3) increases with the ratio of gas mass to total disk mass, $f_{\text {gas }}$, and (4) depends very weakly on the black hole mass, $M_{\mathrm{BH}}$ (Hopkins \& Quataert 2011):

$$
\begin{aligned}
\dot{M}_{\text {Torque }} \approx & \alpha_{\mathrm{T}} f_{\text {disk }}^{5 / 2} \times\left(\frac{M_{\mathrm{BH}}}{10^{8} M_{\odot}}\right)^{1 / 6}\left(\frac{M_{\text {disk }}\left(R_{0}\right)}{10^{9} M_{\odot}}\right)^{1} \\
& \times\left(\frac{R_{0}}{100 \mathrm{pc}}\right)^{-3 / 2}\left(1+\frac{f_{0}}{f_{\text {gas }}}\right)^{-1} M_{\odot} \mathrm{yr}^{-1},
\end{aligned}
$$

where

$$
\begin{gathered}
f_{0} \approx 0.31 f_{\text {disk }}^{2}\left(M_{\text {disk }}\left(R_{0}\right) / 10^{9} M_{\odot}\right)^{-1 / 3}, \\
f_{\text {gas }}\left(R_{0}\right) \equiv M_{\text {gas }}\left(R_{0}\right) / M_{\text {disk }}\left(R_{0}\right),
\end{gathered}
$$

and $\alpha_{\mathrm{T}}$ is a normalization factor of order $\sim 1-10$ that parameterizes the dependence of inflow rates on star formation at scales not resolved. Here we use $\alpha_{\mathrm{T}}=5$ (Hopkins \& Quataert 2011) and evaluate all quantities within a radius $R_{0}=1 \mathrm{kpc}$ that is well resolved in our simulations. The total disk mass $\left(M_{\text {disk }}\right)$ is calculated from kinematic decomposition (similar to $f_{\text {bulge }}$ in Section 3.2), and the total disk mass fraction is calculated as $f_{\text {disk }}=M_{\text {disk }} /\left(M_{\text {gas }}\left(R_{0}\right)+M_{\text {star }}\left(R_{0}\right)\right)$, with $M_{\text {gas }}\left(R_{0}\right)$ and $M_{\text {star }}\left(R_{0}\right)$ the total gas and stellar masses within $R_{0}$, respectively.

Figure 5 shows the evolution of $M_{\text {disk }}, f_{\text {disk }}$, and $f_{\text {gas }}$ for galaxies g54 and g222, as well as the resulting black hole accretion rates according to the gravitational torque model (evaluated for a constant black hole mass $M_{\mathrm{BH}}=10^{5} M_{\odot}$, as before). In our simulations, relative changes in the gravitational torque rate primarily follow from the evolution of $M_{\text {disk }}$ and are, therefore, very sensitive to the morphology of the inner region of the galaxy. The ratio of the gas mass to the total disk mass, $f_{\text {gas }}$, tends to decrease with time as gas is transformed into stars and the disk mass increases, except during galaxy interactions that cause a temporary increase in gas mass and bulge fraction within $R_{0}$ (e.g., galaxy g222 at $\sim 1500$ and $1900 \mathrm{Myr}$ ). Provided that $f_{\text {gas }}>f_{0}$, as it is the case in our simulations down to $z=$ 2 , the gravitational torque rate is fairly insensitive to the gas fraction, since densities at small scales are set by an equilibrium 

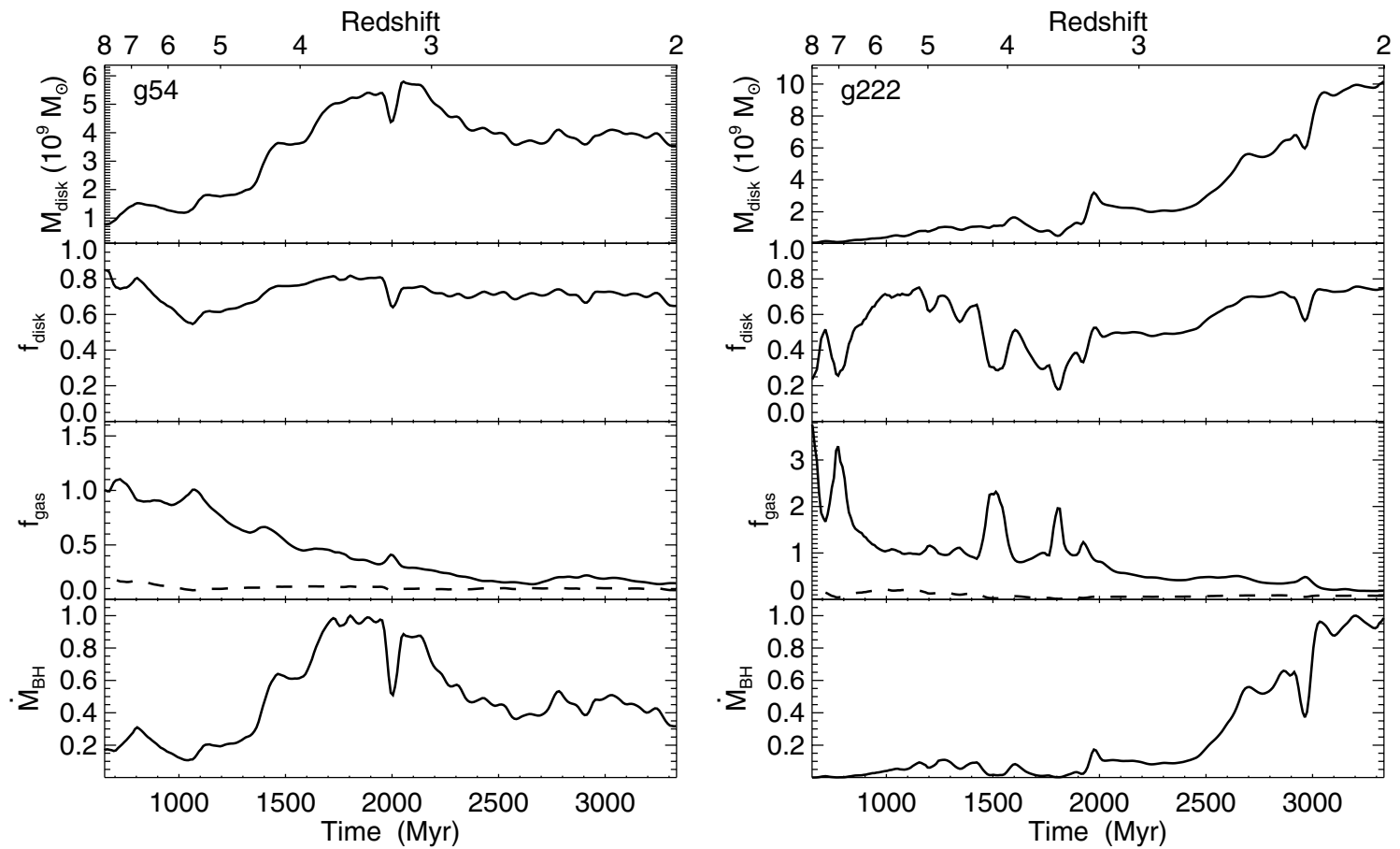

Figure 5. Inferred gravitational torque rates for galaxies g54 (left) and g222 (right). From top to bottom: (1) total (stellar and gas) disk mass evaluated within $R_{0}=1 \mathrm{kpc},(2)$ total disk mass fraction within $R_{0},(3)$ ratio of gas mass to total (stellar and gas) disk mass evaluated at $R_{0}$ (solid line), provided that $f_{\text {gas }} \geqslant f_{0}$ (dashed line) inflow rates are not limited by gas supply, and (4) inferred black hole accretion rates using the analytic model of Hopkins \& Quataert (2011) (Equation (2)), where we have used a constant black hole mass $M_{\mathrm{BH}}=10^{5} M_{\odot}$ at all times and normalized to the peak accretion rate.

between gas inflows and star formation (Hopkins \& Quataert 2011).

The total disk mass within the inner kiloparsec of galaxy g54 increases by a factor of $\sim 5$ from $z=6$ to $z=3$. Most of the accreted gas settles onto a rotationally supported disk from which stars form, resulting in a significant decrease in the stellar mass fraction in a spherical component $\left(f_{\text {bulge }}\right.$; Figure 3$)$. Interestingly, galaxy mergers between $z \approx 3$ and 6 seem to increase the disk mass and therefore the black hole accretion rate, except for a temporary decrease in disk mass at the end of the last merger $(t \sim 2000 \mathrm{Myr})$ that results in a reduction of black hole accretion that lasts $\sim 100 \mathrm{Myr}$, until the disk fraction rises again.

So far, we inferred accretion rates for a constant black hole mass at all times; therefore, it is not possible to provide a direct comparison between the Bondi and gravitational torque models. Despite this, the relative changes in black hole accretion as predicted by the two models seem roughly consistent for galaxy g54, with significant increases in black hole accretion rates correlating with galaxy mergers. However, this scenario changes when looking at the evolution of galaxy g222: mergers at times $t \sim 1500$ and 1900 Myr cause a significant increase in Bondi rates (due to the increase in central density; Figure 4) but not in the gravitational torque rates. In this case, galaxy mergers inhibit the formation of a massive disk component within the inner kiloparsec. The bulge gas fraction increases significantly for a period of $\sim 100-200 \mathrm{Myr}$ after each merger, from which stars quickly form without contributing to the disk component (Figure 3). It is not until the end of the second merger that the disk mass increases for both the gas and stellar components and the black hole accretion rate increases according to the gravitational torque model. Interestingly, the last two mergers at times $t \sim 2700$ and $3000 \mathrm{Myr}$ favor the rapid formation of a massive disk component with the consequent increase in black hole accretion rates.
In summary, galaxy mergers always cause an increase in the Bondi rate due to the increase in density at the center of galaxies, but the effects of mergers on the gravitational torque rates depend on whether they cause an increase or a decrease in the disk mass fraction. Large-scale gravitational torques produced by mergers have the potential to remove angular momentum and drive gas inflows toward the centers of galaxies, as we have seen for two of our simulated galaxies. However, if a merger results in the overall reduction of the disk component, further gravitational instabilities in the disk are suppressed, with the consequent reduction in gas inflow rates. The gravitational torque model yields, therefore, a less direct correspondence between major merger events and rapid phases of black hole growth, which may help explain observational studies showing that active galaxies do not preferentially show merger signatures at $z \sim 2$ (Kocevski et al. 2012; Mullaney et al. 2012b; Schawinski et al. 2012) and at lower redshifts (Gabor et al. 2009; Cisternas et al. 2011b; Böhm et al. 2013).

\section{CONSTRAINTS ON BLACK HOLE GROWTH}

Accretion rates as predicted by the Bondi and gravitational torque models depend on the effects of galaxy interactions and mergers on the evolution of central galaxies. More importantly, these two models have a very different dependence on black hole mass. Thus, in order to provide a more meaningful comparison between these models and get a better intuition on the physical processes governing cosmological black hole growth, they should be compared for a black hole that is growing with time in some consistent way.

If we assume that the observed $z=0$ black hole-galaxy correlations are universal and do not depend strongly on redshift, then we can get a reasonable estimate of black hole masses in terms of the properties of each simulated galaxy. Recent observations of active galaxies indicate a possible evolution of the 


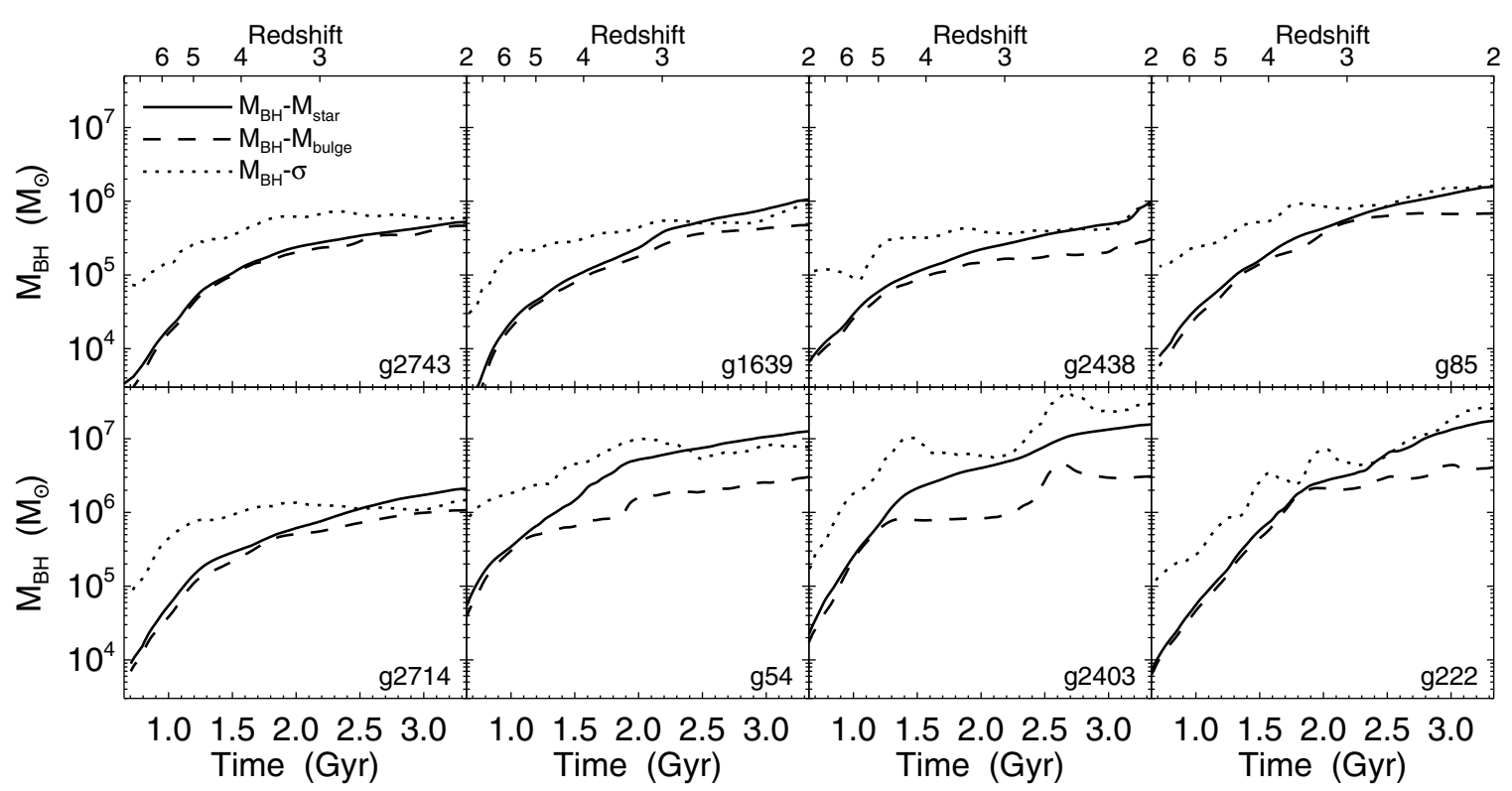

Figure 6. Inferred masses of central black holes as a function of time for all simulated galaxies assuming that the observed $z=0$ black hole-galaxy correlations hold at all times. For each galaxy, black hole masses are calculated according to (1) the $M_{\mathrm{BH}}-\sigma$ relation (Tremaine et al. 2002) for the stellar velocity dispersion within the effective radius (dotted line) and (2) the $M_{\mathrm{BH}}-M_{\text {bulge }}$ relation (Häring \& Rix 2004) for the total stellar mass (solid line) and the bulge mass (dashed line) within the effective radius. For each galaxy, black hole masses have been averaged over timescales of $\sim 200 \mathrm{Myr}$.

$M_{\mathrm{BH}}-M_{\text {bulge }}$ (and perhaps $M_{\mathrm{BH}}-\sigma$ ) relation with redshift (e.g., Decarli et al. 2010; Merloni et al. 2010). Therefore, black hole masses derived from the black hole-galaxy correlations should be taken just as a convenient parameterization as a function of time. In this way, we can infer the absolute instantaneous accretion rates predicted by the Bondi and gravitational torque models and evaluate the implications of different physical processes in the evolution of black holes over cosmic time.

Figure 6 shows the mass of the central black hole as a function of time calculated according to the $M_{\mathrm{BH}}-\sigma$ relation (Tremaine et al. 2002) and the $M_{\mathrm{BH}}-M_{\text {bulge }}$ relation (Häring \& Rix 2004) for all simulated galaxies. Here, we calculate the effective radius of each galaxy $\left(R_{\text {eff }}\right)$ as the two-dimensional projected radius enclosing one-half of the total stellar mass. The galaxy velocity dispersion $(\sigma)$ is, then, evaluated as the one-dimensional stellar velocity dispersion within $R_{\text {eff }}$. Both $R_{\text {eff }}$ and $\sigma$ are averaged over 100 random lines of sight. The bulge mass is calculated as the stellar mass in a spherical component within $R_{\text {eff }}$ (from kinematic decomposition; see Section 3.2) and also taken as the total stellar mass within $R_{\text {eff }}$ for simplicity. Black hole masses shown in Figure 6 have been averaged over time intervals of $\sim 200 \mathrm{Myr}$.

Black hole masses derived from the $M_{\mathrm{BH}}-\sigma$ relation are particularly noisy even after smoothing over long timescales due to the finite resolution and the calculation of $\sigma$ from individual particle motions. We note that the stellar bulge masses inferred from kinematic decomposition usually represent lower limits to the "true" bulge mass, and it is therefore not surprising that black hole masses decrease with time according to the $M_{\mathrm{BH}}-M_{\text {bulge }}$ relation during certain periods of galaxy evolution. Despite this and given the inherently complex evolution of simulated galaxies, it is encouraging that the $M_{\mathrm{BH}}-\sigma$ and $M_{\mathrm{BH}}-M_{\text {bulge }}$ relations predict black hole masses that are largely consistent with each other.

We can now compare the Bondi and gravitational torque models over cosmic time by re-evaluating Equations (1) and (2) for a black hole mass growing according to the black hole-galaxy correlations. Figure 7 shows the evolution of the Bondi and gravitational torque rates, as well as the corresponding Eddington rates, for all simulated galaxies. For simplicity, black hole masses have been taken from the $M_{\mathrm{BH}}-M_{\text {bulge }}$ relation for the total stellar mass within $R_{\text {eff }}$ (solid lines in Figure 6). We note that black hole masses are fixed by the stellar mass of the parent galaxy over time and therefore the inferred Bondi, gravitational torque, and Eddington rates shown here represent instantaneous accretion rates at a given time and do not reflect the actual growth of black holes according to each model.

Some general trends can be identified in Figure 7. At very early times, black holes are small (with masses in the range $\sim 10^{3}-10^{5} M_{\odot}$ according to the $M_{\mathrm{BH}}-M_{\text {bulge }}$ relation of simulated galaxies), and this results in a significant suppression of Bondi rates due to the strong dependence on black hole mass $\left(\propto M_{\mathrm{BH}}^{2}\right)$. The Eddington rate increases linearly with black hole mass; therefore, the inferred instantaneous Bondi rate may eventually become significantly higher than the corresponding Eddington limit for sufficiently massive black holes. In contrast, the gravitational torque model has a very weak dependence on black hole mass $\left(\propto M_{\mathrm{BH}}^{1 / 6}\right)$ and predicts gas inflows from galactic scales to sub-parsec scales that can exceed the Eddington limit by an order of magnitude at very early epochs. The gravitational torque rate also increases with black hole mass but is significantly more sensitive to the evolution of galaxy properties. According to this model, black holes would experience phases of both super-Eddington and sub-Eddington feeding from $z=$ 8 to $z=2$.

The dependence of accretion models on black hole mass can have profound consequences on the inferred black hole evolution. If black hole growth is limited by the gravitational capture of gas as in Bondi, black holes will accrete gas at very low rates early on and may never reach the conditions for critical Eddington growth (e.g., Pelupessy et al. 2007). The rapid early growth of supermassive black holes implied by observations of $z>6$ quasars (Fan et al. 2003; Mortlock et al. 2011), then, requires the formation of massive black hole seeds $\left(\sim 10^{5} M_{\odot}\right)$ 


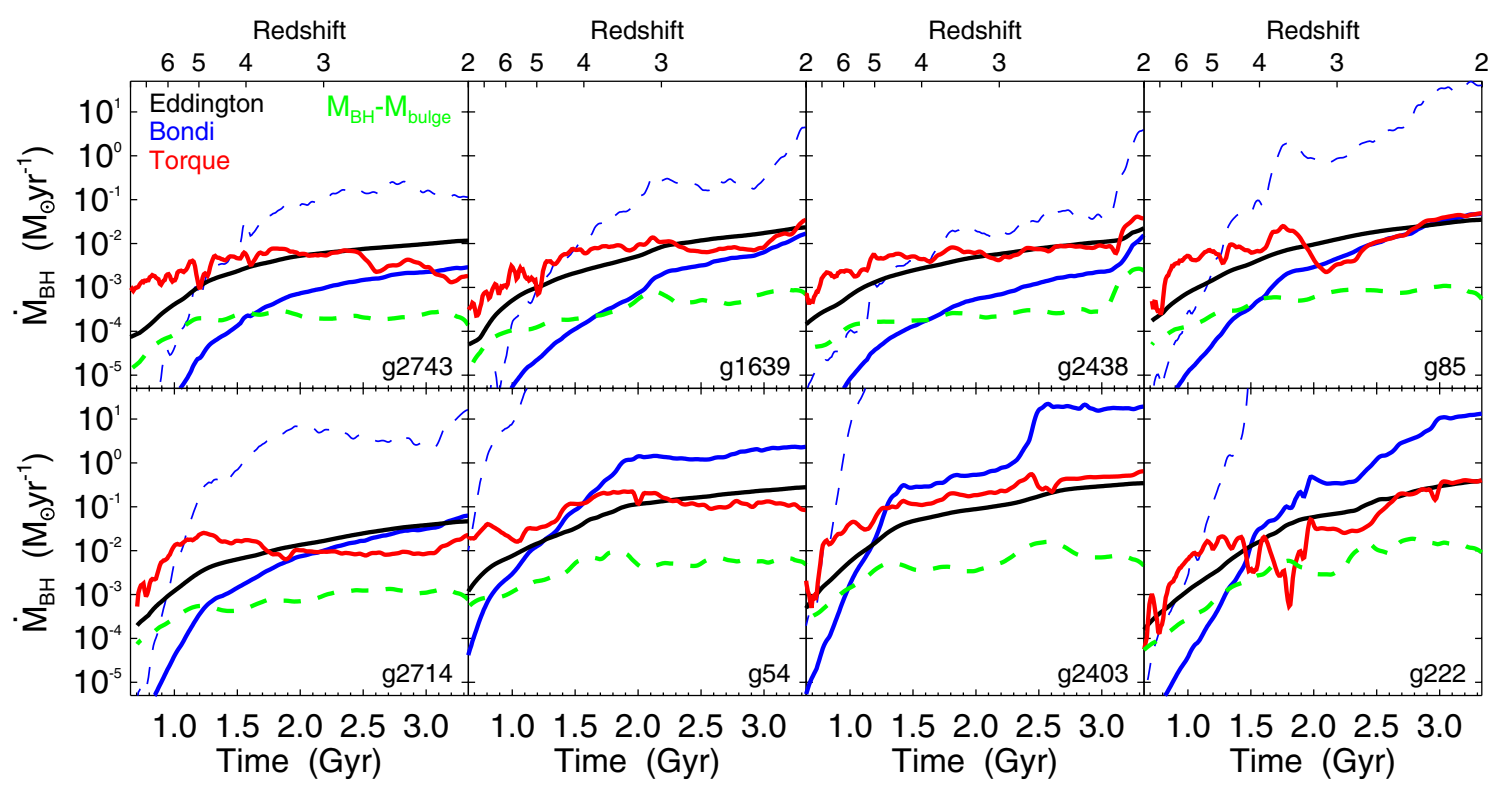

Figure 7. Inferred accretion rates as a function of time for a black hole located at the center of each simulated galaxy. Black hole masses are taken from the $M_{\mathrm{BH}}-M_{\mathrm{bulge}}$ relation for the total stellar mass within the effective radius at all times (Figure 6, solid line), and accretion rates are calculated based on the properties of the host galaxy over time, according to (1) the Bondi-Hoyle-Lyttleton parameterization with $\alpha=100$ (solid blue line) and for the density-dependent $\alpha$ introduced by Booth \& Schaye (2009, dashed blue line), (2) the gravitational torque model of Hopkins \& Quataert (2011, red line), and (3) the Eddington rate (black line). Dashed green lines correspond to the actual accretion rates required for black holes to grow according to the $M_{\mathrm{BH}}-M_{\text {bulge }}$ relation for each galaxy at all times (i.e., the time derivative of black hole masses shown in Figure 6).

(A color version of this figure is available in the online journal.)

for which early accretion is less suppressed (e.g., Di Matteo et al. 2008). Once black holes are massive enough, the Bondi model results in accretion rates well above the Eddington rate and an additional mechanism regulating black hole growth is required.

The black hole mass at which the Bondi rate transitions from being sub-Eddington to super-Eddington depends on model parameters and technical implementations (see, e.g., Booth \& Schaye 2009; Choi et al. 2012). We illustrate this in Figure 7 by showing how the density-dependent boost factor $\alpha \propto \rho^{2}$ results in accretion rates that may differ by several orders of magnitude compared to the constant $\alpha=100$ model, shifting the transition from sub- to super-Eddington to earlier times and, therefore, to lower black hole masses. Despite this, the strong dependence of Bondi rates on the black hole mass has clear consequences at the low-mass and high-mass limits regardless of the particular value of $\alpha$ or other implementation details.

If black hole growth is limited primarily by the transport of angular momentum in the galactic disk, early growth can be rapid, since the inflow rates predicted by the gravitational torque model can be well above the Eddington limit even for small initial black holes. Gas inflows are driven by global gravitational instabilities in the disk and therefore do not depend strongly on black hole mass. Thus, the gravitational torque model may ease constraints on models of black hole seed formation (Volonteri 2010).

Figure 7 also shows the actual accretion rates required for black holes to grow according to the $M_{\mathrm{BH}}-M_{\text {bulge }}$ relation for each galaxy at all times $\left(\dot{M}_{\text {scl }}\right)$. Here we evaluate $\dot{M}_{\text {scl }}$ numerically as the time derivative of the black hole mass calculated from the $M_{\mathrm{BH}}-M_{\text {bulge }}$ relation for each galaxy as a function of time (Figure 6). We find that the inferred Bondi rates are significantly lower than $\dot{M}_{\text {scl }}$ at very early times and become significantly higher than $\dot{M}_{\text {scl }}$ toward $z=2$ for all simulated galaxies. This is a consequence of the strong dependence on black hole mass, as discussed above. In contrast, the gravitational torque rate seems to follow the same dependence as $\dot{M}_{\text {scl }}$, but it is about an order of magnitude higher at all times. If black hole growth is limited primarily by the transport of angular momentum by gravitational instabilities, it is, therefore, not expected that all of the mass that is fed into the accretion flow at $\lesssim 0.01 \mathrm{pc}$ scales finds its way down to the central black hole. In fact, a number of theoretical and observational studies have shown that only a small fraction of the mass inflow is retained in the accretion flow, with the rest lost to winds and outflows (e.g., Di Matteo et al. 2000; King et al. 2013; Yuan et al. 2012).

We compare in Figure 8 the inferred Bondi rates (with $\alpha=100)$ and gravitational torque rates to $\dot{M}_{\mathrm{scl}}$ for all galaxies at all times. Remarkably, a simple power law can provide a reasonable fit for all simulated galaxies. Specifically, we find

$$
\begin{gathered}
\dot{M}_{\text {Bondi }}=(6.07 \pm 0.06) \dot{M}_{\mathrm{scl}}^{(2.73 \pm 0.02)}, \\
\dot{M}_{\text {Torque }}=(1.26 \pm 0.03) \dot{M}_{\mathrm{scl}}^{(1.004 \pm 0.010)} .
\end{gathered}
$$

We note that, in most cases, large deviations from the best fit are due to the calculation of black hole masses (and therefore $\dot{M}_{\text {scl }}$ ) directly from the evolution of galaxy masses. The large power index $(\sim 2.7)$ for the Bondi rate confirms the trends found in Figure 7 for all simulated galaxies: Bondi rates evolve from being significantly lower than $\dot{M}_{\text {scl }}$ at small black hole masses to being significantly higher at large black hole masses. That is, black holes growing at the Bondi rate either would lie well below the $M_{\mathrm{BH}}-M_{\text {bulge }}$ relation at all times or would become overly massive by $z=2$. If the Bondi rate provides a good estimate of the true accretion rate, the large power index in Equation (5) suggests that (1) the initial black hole mass and the boost factor $\alpha$ need to be set to allow for early growth and (2) some form of feedback that becomes more efficient at higher accretion rates is required. Indeed, suitable choices of 


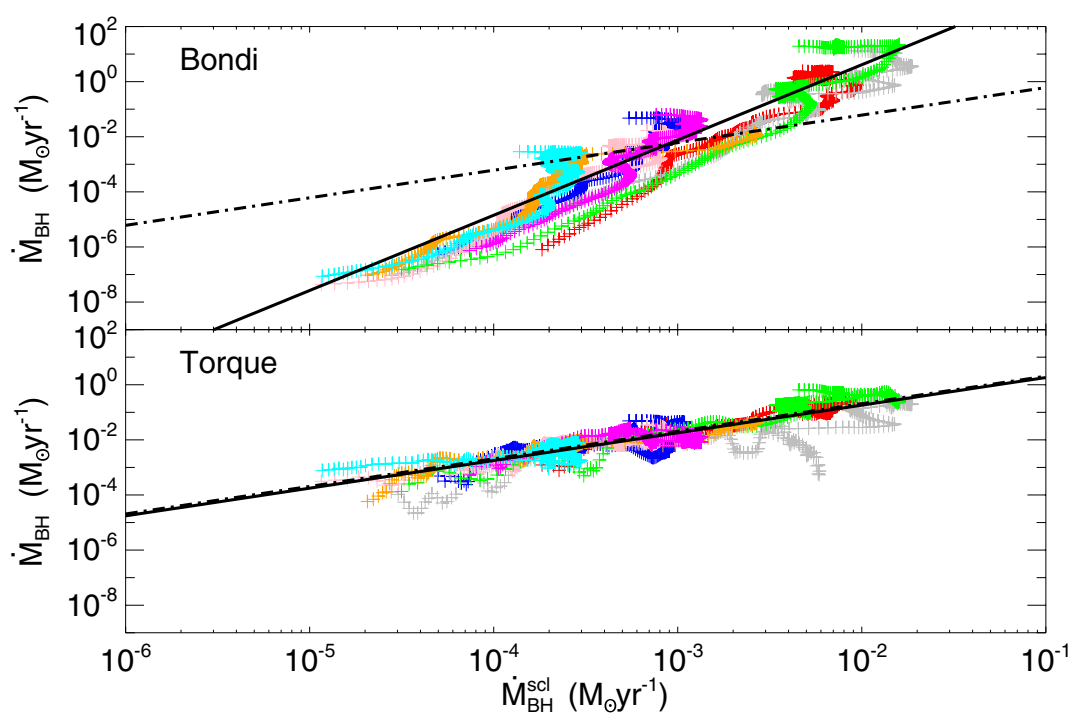

Figure 8. Bondi rates with $\alpha=100$ (top) and gravitational torque rates (bottom) as a function of the accretion rate required for black holes to grow according to the $M_{\mathrm{BH}}-M_{\text {bulge }}$ relation for each galaxy at all times $\left(\dot{M}_{\mathrm{scl}}\right)$. Each color corresponds to a different galaxy as in Figure 2 . Solid lines show the best power-law fit for each model, and dash-dotted lines correspond to a linear relation with constant slope given by median $\left(\dot{M}_{\text {Bondi }} / \dot{M}_{\text {scl }}\right)$ (top) and median $\left(\dot{M}_{\text {Torque }} / \dot{M}_{\text {scl }}\right)($ bottom) for all galaxies at all times.

(A color version of this figure is available in the online journal.)

model parameters and the coupling of a fraction of the accretion luminosity to the surrounding gas in the form of thermal energy have been successful in reproducing the observed black hole-galaxy correlations (Di Matteo et al. 2005; Robertson et al. 2006; Hopkins et al. 2007; Booth \& Schaye 2009).

The gravitational torque rates are, on average, directly proportional to the accretion rates required by the $M_{\mathrm{BH}}-M_{\text {bulge }}$ relation, with a power index very close to unity (Equation (6)). Indeed, a linear relation $\dot{M}_{\text {Torque }}=\epsilon_{\mathrm{m}}^{-1} \dot{M}_{\text {scl }}$ with constant slope given by $\epsilon_{\mathrm{m}}^{-1}=\operatorname{median}\left(\dot{M}_{\text {Torque }} / \dot{M}_{\text {scl }}\right)$ for all galaxies at all times provides a very reasonable fit to the numerical results (Figure 8). Remarkably, a simple mass retention rate in the accretion flow, $\epsilon_{\mathrm{m}} \approx 5 \%$, seems sufficient to bring the gravitational torque rates down to $\dot{M}_{\text {scl }}$ over a range of more than three orders of magnitude in accretion rates, for all simulated galaxies, and at all times. Therefore, the additional mechanism required to regulate black hole growth according to the $M_{\mathrm{BH}}-M_{\text {bulge }}$ relation has to be roughly equally efficient regardless of the accretion rate. Since the radiative luminosity is proportional to the black hole accretion rate, the gravitational torque model seems to disfavor AGN feedback acting at galactic scales as the primary mechanism regulating black hole growth.

\section{TORQUE-LIMITED GROWTH}

The transport of angular momentum in the galactic disk is a required process for black hole growth regardless of the presence and effects of AGN feedback. Therefore, we can explore an alternative scenario in which winds and outflows from the inner accretion disk do not affect the accretion flow and simply result in the reduction of black hole accretion rates by a constant mass retention rate $\epsilon_{\mathrm{m}}$. The gravitational torque model describes gas inflows from galactic scales down to $<0.1 \mathrm{pc}$ scales, and therefore it can be interpreted as the mass feeding rate into the black hole accretion disk. Some of this mass may be lost by radiatively driven outflows and other processes (e.g., Proga et al. 2000; Ohsuga et al. 2005; Yuan et al. 2012), while a small fraction reaches the central black hole. In this scenario, the central black hole grows on average at a fraction $\epsilon_{\mathrm{m}}$ of the

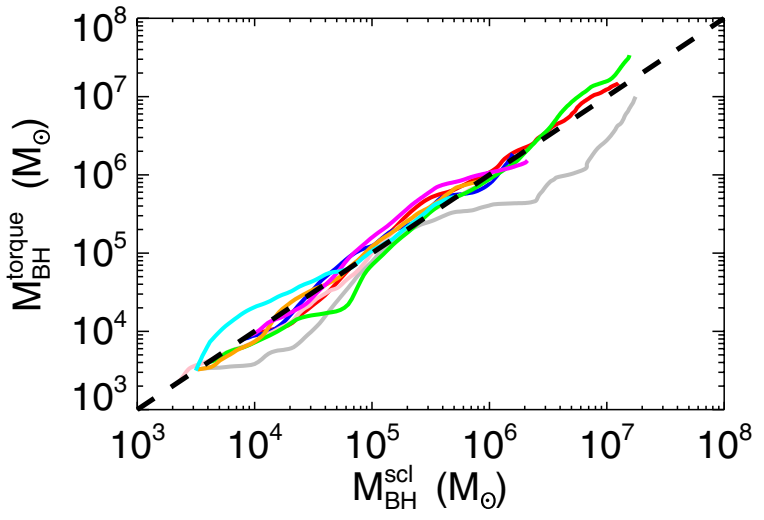

Figure 9. Black hole mass calculated self-consistently from the gravitational torque rate corrected by the mass retention rate $\epsilon_{\mathrm{m}}=0.05$ (by integration of Equation (7)) as a function of black hole mass derived from the $M_{\mathrm{BH}}-M_{\text {bulge }}$ relation for each galaxy at all times. Each color corresponds to a different galaxy as in Figure 2.

(A color version of this figure is available in the online journal.)

large-scale gas inflow rate:

$$
d M_{\mathrm{BH}} / d t=\epsilon_{\mathrm{m}} \dot{M}_{\text {Torque }}(t) .
$$

Figure 9 shows the evolution of black hole masses predicted by the gravitational torque model normalized by the mass retention rate $\epsilon_{\mathrm{m}} \approx 0.05\left(M_{\mathrm{BH}}^{\text {Torque }}\right)$, as a function of the black hole mass obtained from the $M_{\mathrm{BH}}-M_{\text {bulge }}$ relation for each galaxy at each time step $\left(M_{\mathrm{BH}}^{\mathrm{scl}}\right)$. Here, we are simply integrating Equation (7) for an initial black hole mass that is consistent with the $M_{\mathrm{BH}}-M_{\text {bulge }}$ relation for each galaxy. We note that $\dot{M}_{\text {Torque }}(t)$ is self-consistently calculated based on the morphological properties of each galaxy over time (Equation (2)) and updated with the appropriate black hole mass at each time step, as given by Equation (7). Remarkably, we find that black holes evolve approximately along the $M_{\mathrm{BH}}^{\text {Torque }}=M_{\mathrm{BH}}^{\text {scl }}$ line and therefore remain consistent with the $M_{\mathrm{BH}}-M_{\text {bulge }}$ relation. 


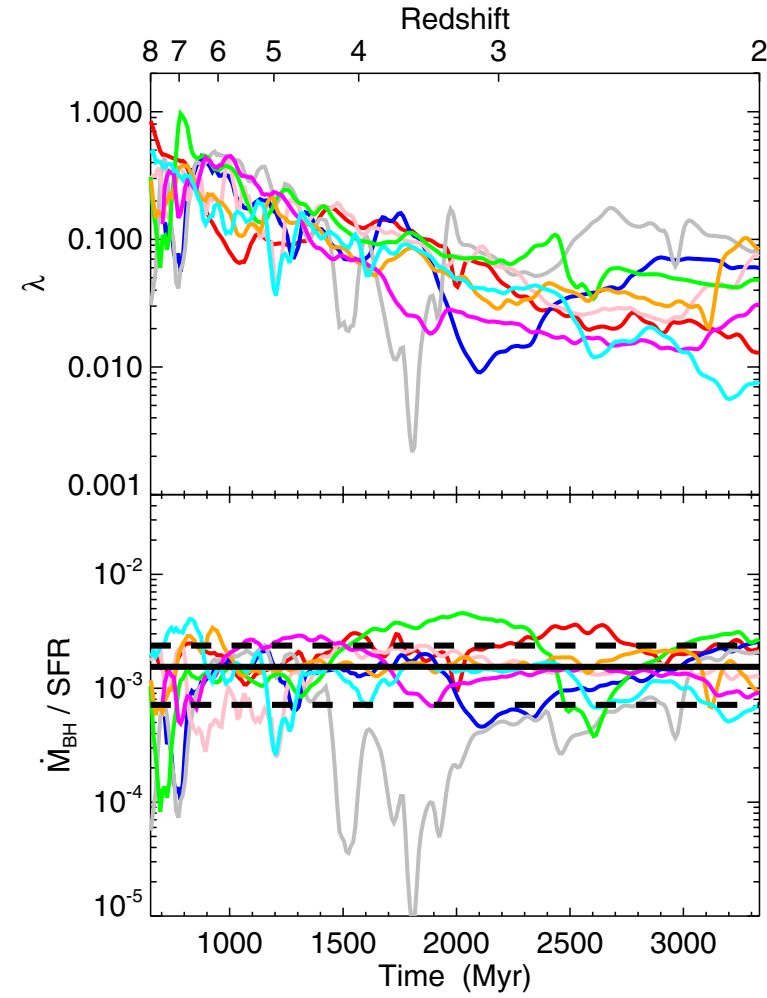

Figure 10. Top: Eddington ratio (defined here as $\dot{M}_{\mathrm{BH}} / \dot{M}_{\mathrm{Edd}}$ ) for black holes growing self-consistently at the gravitational torque rate corrected by the mass retention rate $\epsilon_{\mathrm{m}}=0.05$ (by integration of Equation (7)) for each galaxy as a function of time. Bottom: ratio of the black hole accretion rate to the total SFR within the effective radius. Black solid and dashed lines show a running average of $\dot{M}_{\mathrm{BH}} / \mathrm{SFR}$ over all galaxies and all times and the standard deviation, respectively. Initial black hole masses are taken to be consistent with the $z=0$ $M_{\mathrm{BH}}-M_{\text {bulge }}$ relation (Figure 6). Each color corresponds to a different galaxy as in Figure 2.

(A color version of this figure is available in the online journal.)

In Section 7.2, we show that $\epsilon_{\mathrm{m}}$ sets the normalization of the $M_{\mathrm{BH}}-M_{\text {bulge }}$ relation. We note, however, that the gravitational torque model (Equation (2)) contains a normalization constant of order $\alpha_{\mathrm{T}} \approx 1-10$ that parameterizes the effects of nuclear star formation on black hole accretion (Hopkins \& Quataert 2011). Therefore, the normalization of the $M_{\mathrm{BH}}-M_{\text {bulge }}$ relation is actually determined by the product $\alpha_{\mathrm{T}} \epsilon_{\mathrm{m}}$. Given that plausible values of these two constants differ by about two orders of magnitude, we choose to interpret them as parameterizing different physical processes: $\alpha_{\mathrm{T}}$ controls how much gas is converted into stars versus feeding the black hole accretion disk (Hopkins \& Quataert 2011), and $\epsilon_{\mathrm{m}}$ controls what fraction of the mass feeding the accretion disk is finally accreted by the black hole. We implicitly assume that the remaining mass, a fraction $\left(1-\epsilon_{\mathrm{m}}\right)$ of the disk feeding rate, is expelled in the polar direction without significantly halting further accretion.

We can now look back at the accretion histories of black holes growing according to the gravitational torque model with the appropriate normalization $\left(\epsilon_{\mathrm{m}} \approx 0.05\right)$. Figure 10 shows the evolution of the inferred Eddington ratios of black holes located at the center of all simulated galaxies. Despite the large scatter between galaxies and the high temporal variability, there is a clear trend for Eddington ratios to decrease from $\sim 0.1-1$ at $z=$ 8 down to $\sim 0.01-0.1$ at $z=2$. This is in agreement with recent observations of $z \gtrsim 4$ quasars showing that higher redshift black holes have lower masses but are accreting at higher Eddington ratios compared to lower redshift black holes (De Rosa et al. 2011; Trakhtenbrot et al. 2011).

Intriguingly, the inferred Eddington ratios evolve in a manner very similar to the evolution of specific SFRs (Figure 2): both quantities seem to decrease by about a factor of 10 from $z=8 \rightarrow 2$, on average. In fact, Figure 10 (lower panel) shows that the ratio of the black hole accretion rate to the SFR within the effective radius of galaxies is, on average, very close to the ratio of black hole mass to stellar mass as inferred from the local $M_{\mathrm{BH}}-M_{\text {bulge }}$ relation:

$$
\left\langle\frac{\dot{M}_{\mathrm{BH}}}{\mathrm{SFR}}\right\rangle \approx 0.00154 \pm 0.0008
$$

where the average (and standard deviation) has been calculated for all galaxies and all times. There is, of course, large scatter in $\dot{M}_{\mathrm{BH}} / \mathrm{SFR}$ between different galaxies and evolution times, but this ratio is, on average, remarkably flat in the redshift range $z=2-8$ : the bulk of black hole and galaxy growth occurs in tandem, at rates governed by cosmological infall and transport of angular momentum in the galaxy disk, and with no feedbackmediated coupling between black holes and parent galaxies.

The torque-limited model predicts, therefore, a natural connection between AGN activity and SFR of galaxies on cosmological timescales, in agreement with observations showing that the volume-averaged ratio of black hole accretion rate to SFR agrees with the ratio of black hole mass to stellar mass as inferred from the local $M_{\mathrm{BH}}-M_{\text {bulge }}$ relation (Heckman et al. 2004; Zheng et al. 2009). Furthermore, these results are consistent with recent observational evidence for a constant black hole accretion to star formation ratio independent of redshift since $z \sim 2$ (Rafferty et al. 2011; Mullaney et al. 2012a). Since black holes and galaxies evolve approximately along the scaling relations, the torque-limited model provides support for a roughly constant ratio of black hole mass to stellar mass independent of redshift, consistent with the conclusions of Jahnke et al. (2009), Cisternas et al. (2011a), and Mullaney et al. (2012a) at redshifts $z \lesssim 2$. The AGN-SFR connection is, however, not direct in a galaxy-by-galaxy basis and at all times (as evident from the scatter in Figure 10, lower panel), which may help explain the large scatter in observed AGN-to-star-formation ratios in individual systems (e.g., Rafferty et al. 2011).

Torque-limited growth is, therefore, a natural alternative to feedback-regulated models and is indeed consistent with our "feedback-free" starting point, since black hole accretion rates have been inferred based on galaxy properties from simulations with no AGN feedback model (Section 4). AGN feedback may have a significant impact on the host galaxy, but it is not required for regulating black hole growth. Angular momentum transport (in the galaxy as well as the accretion disk), together with competition with star formation, seems to be sufficient for black holes to grow according to the scaling relations from early times down to $z=2$. This is explored in more detail in the next section.

\section{BLACK HOLE-GALAXY CORRELATIONS}

Figure 11 shows how galaxies and black holes evolve in the $M_{\mathrm{BH}}-M_{\text {bulge }}$ and $M_{\mathrm{BH}}-\sigma$ planes according to the torque-limited model (Equation (7)). As we have seen in the previous section, a simple normalization constant $\epsilon_{\mathrm{m}} \approx 0.05$ applied to the gravitational torque model brings black holes and galaxies close to the $M_{\mathrm{BH}}-M_{\text {bulge }}$ relation of Häring \& Rix (2004). Calculation of the galaxy stellar velocity dispersion from particle motions results in higher scatter but still shows that black holes growing 

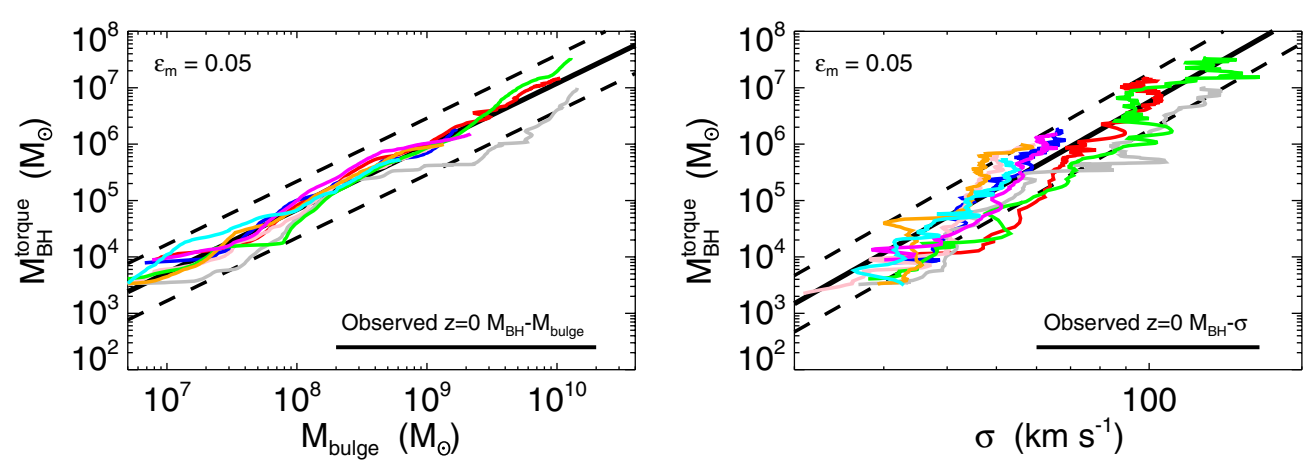

Figure 11. $M_{\mathrm{BH}}-M_{\text {bulge }}$ (left) and $M_{\mathrm{BH}}-\sigma$ (right) relations for black holes growing self-consistently at the gravitational torque rate corrected by the mass retention rate $\epsilon_{\mathrm{m}}=0.05$ (by integration of Equation (7)) for each galaxy at all times. Initial black hole masses are taken to be already consistent with the scaling relations. Black solid lines show the $M_{\mathrm{BH}}-M_{\text {bulge }}$ and $M_{\mathrm{BH}}-\sigma$ relations of Häring \& Rix (2004) and McConnell et al. (2011), respectively, and black dashed lines indicate a 0.5 dex scatter in black hole mass for each relation. The galaxy velocity dispersion $(\sigma)$ is calculated as the one-dimensional stellar velocity dispersion within the effective radius $\left(R_{\text {eff }}\right)$ averaged over 100 random lines of sight. $M_{\text {bulge }}$ is taken as the total stellar mass within $R_{\text {eff }}$. Each color corresponds to a different galaxy as in Figure 2. (A color version of this figure is available in the online journal.)

according to Equation (7) are roughly consistent with the $M_{\mathrm{BH}}-\sigma$ relation of McConnell et al. (2011).

In Section 5 we have inferred "instantaneous" accretion rates according to the Bondi and gravitational torque models by assuming that the observed $M_{\mathrm{BH}}-M_{\text {bulge }}$ relation holds at all times. This was a convenient way of parameterizing black hole mass as a function of time for each galaxy, but there is a priori no reason to think that galaxies and black holes should evolve together at all times. The gravitational torque rates inferred in this way were often of the order of the Eddington limit (Figure 7), and therefore we might be tempted to think that black holes growing at a fixed fraction of the Eddington limit could also grow in a manner consistent with the black hole-galaxy correlations. However, once we release the assumption of black hole-galaxy co-evolution (so that black hole masses are no longer given by the $M_{\mathrm{BH}}-M_{\text {bulge }}$ relation) and calculate black hole growth self-consistently over time, we see that a simple normalization constant cannot compensate for the exponential Eddington growth. The fact that the torquelimited model naturally leads to black holes growing on average according to the black hole-galaxy correlations is a non-trivial consequence of the weak dependence on black hole mass and the strong dependence on galaxy scale properties.

In the remainder of this section, we show how the inferred black hole-galaxy correlations depend on model parameters and implementation details, and we discuss additional implications of the torque-limited model.

\subsection{Initial Black Hole Mass}

We have shown that, provided the initial black hole mass lies approximately in the $M_{\mathrm{BH}}-M_{\text {bulge }}$ relation, the gravitational torque model scaled by a simple constant factor predicts that black holes and galaxies evolve together along the scaling relations. However, black holes do not necessarily know what is the correct initial mass for a given galaxy. In Figure 12, we evaluate how changes in the initial conditions affect black hole growth and the inferred $M_{\mathrm{BH}}-M_{\text {bulge }}$ and $M_{\mathrm{BH}}-\sigma$ relations. First, we grow black holes with initial masses either a factor of 10 above or below the scaling relations for each galaxy. We find that, regardless of the initial mass, black holes tend to evolve onto the observed black hole-galaxy correlations. In our simulations, accretion rates are governed by the disk mass, with a very weak dependence on black hole mass, and therefore black holes with different initial masses grow at comparatively similar rates for a given galaxy. Since most of the black hole mass comes from accretion, which depends mostly on the evolution of the galaxy, the initial conditions are erased and the black hole-galaxy correlations are established.

This is also observed when the initial black hole masses are uncorrelated with their parent galaxies. Figure 12 (middle panels) shows the $M_{\mathrm{BH}}-M_{\text {bulge }}$ and $M_{\mathrm{BH}}-\sigma$ relations when initial black hole masses are taken to be the same for all galaxies and ranging from $10^{2}$ to $10^{5} M_{\odot}$. For simplicity, we show the evolution of the "average galaxy" that we calculate by averaging black hole mass, bulge mass, and velocity dispersion over all galaxies at each time step. Provided we let black holes and galaxies evolve for a sufficient amount of time, they all converge on average toward the scaling relations. Finally, we show in Figure 12 (bottom panels) that this convergence toward the scaling relations is also observed when we take black hole masses for all galaxies at all times and average them within bins in either bulge mass or velocity dispersion regardless of the evolution time of each galaxy.

Black hole-galaxy correlations arising from the torquelimited model are robust to changes in the initial mass of black holes and do not require specific tuning of the mass retention rate $\epsilon_{\mathrm{m}}$. Importantly, black holes with masses as low as $100 M_{\odot}$ at $z=8$ are able to grow comparatively faster than higher mass black holes, opening up possibilities for light seed formation mechanisms, such as remnants of Population III stars, being the progenitors of today's supermassive black holes (Madau \& Rees 2001; Volonteri 2010).

\subsection{Mass Retention Rate}

The mass retention rate $\left(\epsilon_{\mathrm{m}}\right)$ is a free parameter in the torquelimited model that we have introduced in order to match the observed $M_{\mathrm{BH}}-M_{\text {bulge }}$ relation (Sections 5 and 6). Figure 13 shows how the inferred $M_{\mathrm{BH}}-M_{\text {bulge }}$ relation changes when using a wide range of retention values, from an extreme situation in which $99.9 \%$ of the inflow mass is lost in outflows, to the upper limit $\epsilon_{\mathrm{m}}=1$ in which all of the infalling gas from larger scales is accreted by the black hole (which corresponds to the original normalization of Hopkins \& Quataert 2011). As we might expect, we find that $\epsilon_{\mathrm{m}}$ only affects the normalization of the $M_{\mathrm{BH}}-M_{\text {bulge }}$ relation, with little effects on the slope except at very early times.

A somewhat similar torque-limited scenario has been proposed by Escala (2006) and tested with idealized sub-parsec 

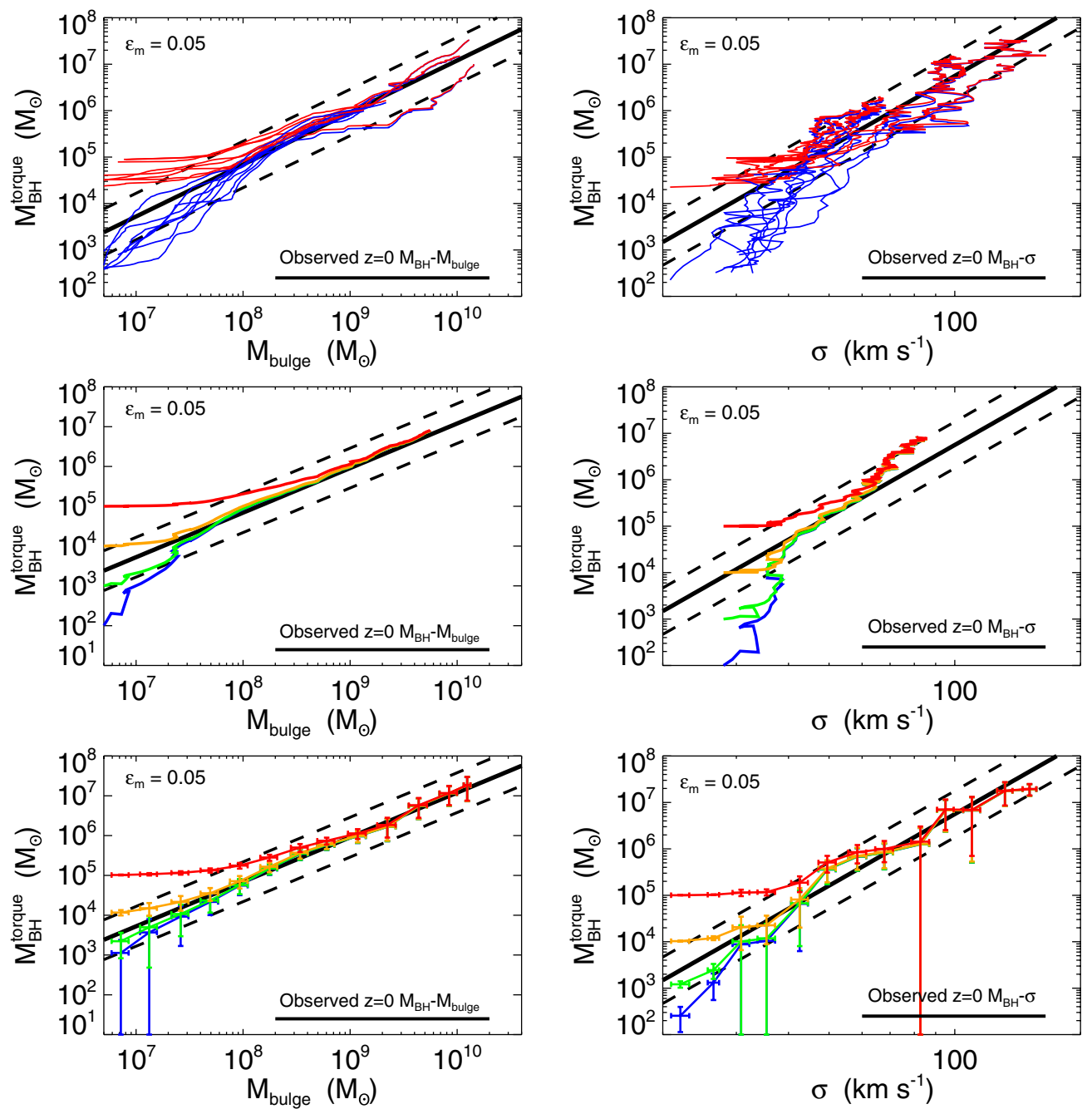

Figure 12. $M_{\mathrm{BH}}-M_{\text {bulge }}$ (left panels) and $M_{\mathrm{BH}}-\sigma$ (right panels) relations for black holes growing self-consistently at the gravitational torque rate (Equation (7) with $\epsilon_{\mathrm{m}}=0.05$ ) for different initial black hole masses. Top panels: the initial black hole mass is taken to be either 10 times higher (red) or 10 times lower (blue) compared to the scaling relation for each galaxy. Middle panels: black hole mass as a function of either bulge mass or velocity dispersion for the "average" galaxy (i.e., averaging $M_{\mathrm{BH}}, M_{\text {bulge }}$, and $\sigma$ over all galaxies at each time step) for initial black hole masses ranging from $10^{2}$ to $10^{5} M_{\odot}$ (from blue to red). Bottom panels: average black hole mass within logarithmically spaced bins in either bulge mass or velocity dispersion, using all simulated galaxies at all time steps, and for initial black hole masses taken from $10^{2}$ to $10^{5} M_{\odot}$ (from blue to red). Error bars show the dispersion in each bin. Black solid and dashed lines correspond to the observed scaling relations (Häring \& Rix 2004; McConnell et al. 2011) and a 0.5 dex scatter in black hole mass, respectively.

(A color version of this figure is available in the online journal.)
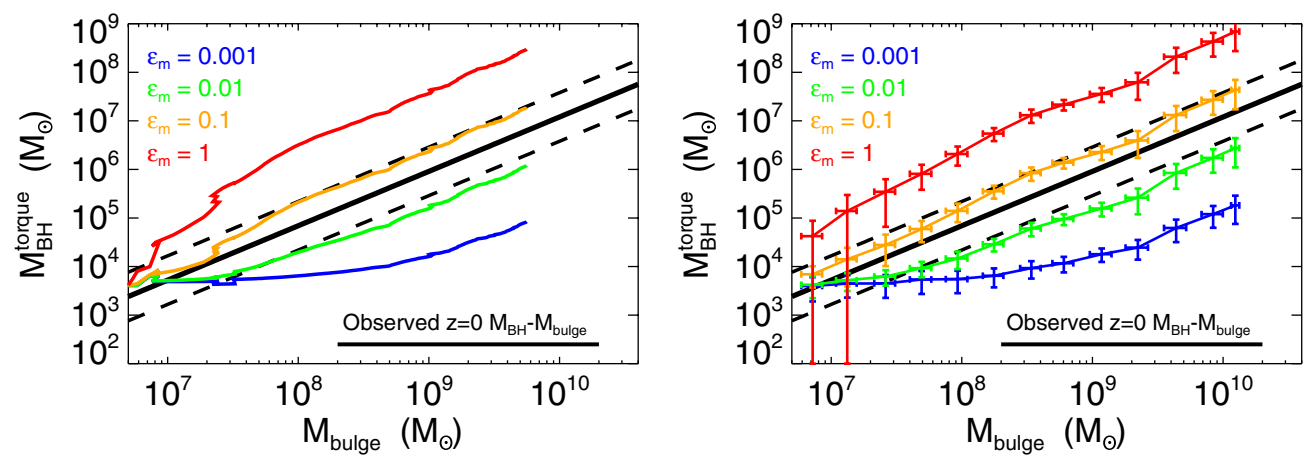

Figure 13. Effects of varying the mass retention rate from $\epsilon_{\mathrm{m}}=0.001$ to $\epsilon_{\mathrm{m}}=1$ by factors of 10 (from blue to red) in the $M_{\mathrm{BH}}-M_{\mathrm{bulge}}$ relation. Left: black hole mass as a function of bulge mass averaged over all galaxies at each time step for initial black hole masses consistent with the $M_{\mathrm{BH}}-M_{\text {bulge }}$ relation. Right: average black hole mass within logarithmically spaced bins in bulge mass for all simulated galaxies, at all time steps, and taking initial black hole masses consistent with the $M_{\mathrm{BH}}-M_{\text {bulge }}$ relation for each galaxy. Black solid and dashed lines correspond to the observed $M_{\mathrm{BH}}-M_{\text {bulge }}$ relation (Häring \& Rix 2004) and a 0.5 dex scatter in black hole mass, respectively.

(A color version of this figure is available in the online journal.) 

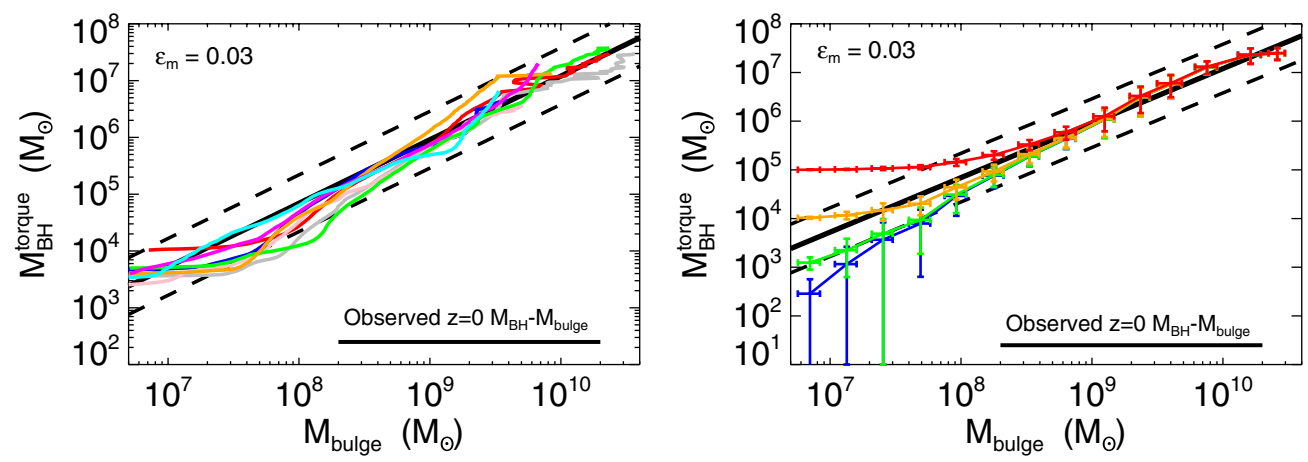

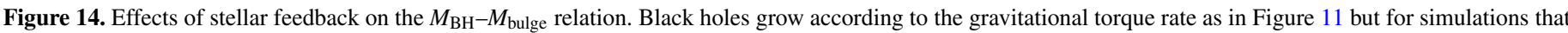

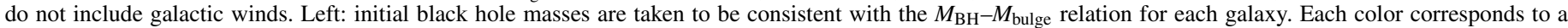

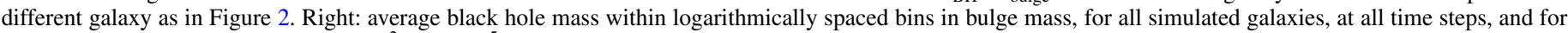

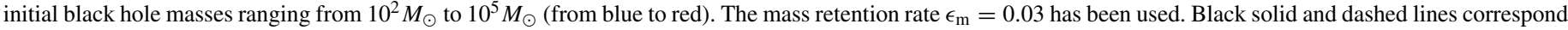
to the observed $M_{\mathrm{BH}}-M_{\text {bulge }}$ relation (Häring \& Rix 2004) and a 0.5 dex scatter in black hole mass, respectively.

(A color version of this figure is available in the online journal.)
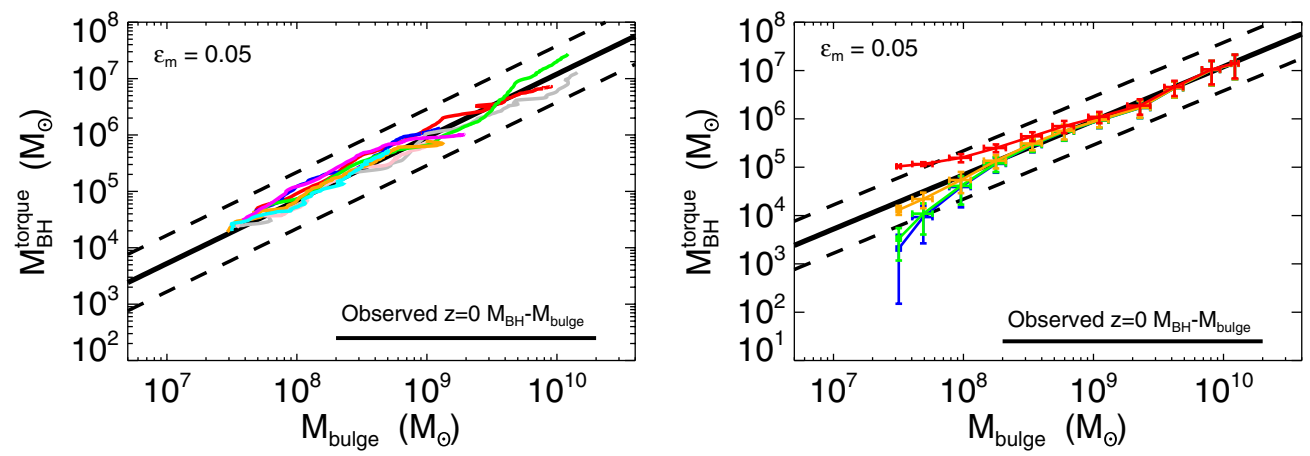

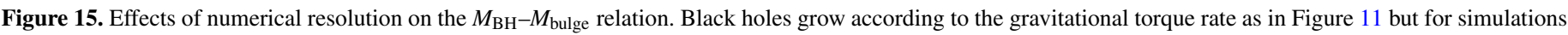

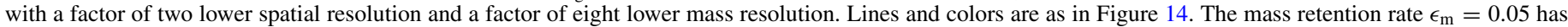
been used.

(A color version of this figure is available in the online journal.)

resolution simulations of a nuclear galactic disk (Escala 2007). They find that a mass retention rate $\epsilon_{\mathrm{m}} \approx 15 \%$ is required in order to match the $M_{\mathrm{BH}}-M_{\text {bulge }}$ relation, similar to our result $\epsilon_{\mathrm{m}} \approx 5 \%$ given that both sets of simulations probe spatial scales and timescales that are substantially different.

\subsection{Stellar Feedback and Resolution Effects}

In a separate work using the same set of simulations, we have shown that strong stellar feedback in the form of galactic outflows can have a significant impact on the star formation, morphological, and kinematic properties of high-redshift galaxies (Anglés-Alcázar et al. 2013). Feedback from star formation could therefore affect black hole growth (e.g., Cen 2012) and possibly the inferred black hole-galaxy correlations.

Figure 14 shows the $M_{\mathrm{BH}}-M_{\text {bulge }}$ relation obtained for the same sample of galaxies but this time from simulations that do not include models for galactic winds. We find that the scaling relations are still reproduced by the torque-limited model and perhaps even with smaller scatter. The convergence toward the $M_{\mathrm{BH}}-M_{\text {bulge }}$ relation for different initial black hole masses is retained (Section 7.1), and similar results are obtained for the $M_{\mathrm{BH}}-\sigma$ relation. Predictions of the gravitational torque model are therefore robust to changes in stellar feedback. Even if galaxies from simulations with no winds become significantly more massive and less gas rich owing to higher SFRs (AnglésAlcázar et al. 2013), black holes still grow according to the scaling relations in the redshift range $z=8 \rightarrow 2$. This suggests that galaxies regulate themselves via an equilibrium between inflows and outflows and black holes grow according to the properties of their host galaxies, at a rate given by the gas supply from galactic scales.

We have also investigated the effects of numerical resolution on the scaling relations. Figure 15 shows the $M_{\mathrm{BH}}-M_{\text {bulge }}$ relation obtained for the same sample of galaxies from simulations carried out with a factor of two lower spatial resolution and a factor of eight lower mass resolution (including galactic outflows). Since we require galaxies to contain at least 100 gas particles and 100 star particles within $R_{0}=1 \mathrm{kpc}$ in order to resolve their central morphology, these lower resolution simulations can follow the evolution of black holes only after their parent galaxies are a factor of $\sim 8$ more massive compared to the high-resolution simulations. Despite the reduced resolution, the $M_{\mathrm{BH}}-M_{\text {bulge }}$ relation arising from the gravitational torque model shows good numerical convergence.

We note that the values of $\epsilon_{\mathrm{m}}$ obtained for simulations with no galactic outflows and the low-resolution simulations are slightly different from our fiducial runs, but they are all consistent with a typical mass retention rate of a few percent of the gas supply.

\subsection{Black Hole Mergers}

It has been shown recently that black hole-galaxy correlations may be a natural consequence of hierarchical structure formation, so that an initially uncorrelated distribution of black hole and stellar masses tends to converge toward the scaling 
relations through successive mergings of black holes and galaxies (Peng 2007; Jahnke \& Macciò 2011). In this scenario, AGN feedback and self-regulated growth are not required, but it is assumed that galaxy mergers always result in the merging of their central black holes. Here, we have shown how torque-limited growth yields black hole-galaxy correlations regardless of the initial masses of black holes and galaxies, and also without the need for AGN feedback and self-regulated growth. We have, however, left off the discussion of the role of black hole mergers in the overall black hole growth. Here, we assume that most of the black hole mass comes from accretion (Soltan 1982) and simply neglect the mass contribution from mergers. Given that only a fraction of mass in our galaxies comes from major mergers (Murali et al. 2002; Keres et al. 2005), we do not expect a significant shift on the inferred black hole-galaxy correlations due to black hole mergers.

\subsection{Evolution of the Scaling Relations}

Recent observations of active galaxies seem to indicate an evolution of the $M_{\mathrm{BH}}-M_{\text {bulge }}$ (and perhaps $M_{\mathrm{BH}}-\sigma$ ) relation with redshift, with black holes being more massive for a given galaxy mass (or velocity dispersion) at higher redshifts compared to the local relations (Shen et al. 2008; Woo et al. 2008; Decarli et al. 2010; Greene et al. 2010; Merloni et al. 2010). There are also findings of no significant evolution (Jahnke et al. 2009) and potentially even undermassive black holes in $z \sim 2$ infraredselected galaxies (Shapiro et al. 2009). In either case, evaluations of the redshift evolution of the black hole scaling relations may be biased by selection effects (Lauer et al. 2007).

In the context of torque-limited growth, we find no evidence for evolution of the scaling relations at least down to $z=2$, since black holes and galaxies seem to converge toward the scaling relations regardless of their initial masses, provided $\alpha_{\mathrm{T}}$ and $\epsilon_{\mathrm{m}}$ do not evolve with redshift and are well represented by a constant factor. Therefore, significant deviations at early times could in principle reflect the "initial conditions" for co-evolution of black holes and galaxies (Figure 12).

We note that $\epsilon_{\mathrm{m}}$ has been fixed here to match the $M_{\mathrm{BH}}-M_{\text {bulge }}$ relation of Häring \& Rix (2004) for the total stellar mass within the effective radius rather than the stellar bulge mass. Given that our galaxies contain a significant stellar disk component at $z=2$ (Anglés-Alcázar et al. 2013), it is plausible that there is significant evolution of the $M_{\mathrm{BH}}-M_{\text {bulge }}$ relation with respect to bulge mass but not with respect to total stellar mass, as suggested by Jahnke et al. (2009). If most of the mass of bulges and elliptical galaxies today comes from redistribution of the stellar bulge+disk of $z=2$ galaxies (through mergers or other processes), no further black hole accretion would be required to match the local scaling relations. We speculate that the formation of compact bulges would indeed decrease the gravitational torque rate (since it is proportional to the disk mass), truncating significant black hole growth at late times.

\section{SUMMARY AND CONCLUSIONS}

We have used cosmological zoom simulations of galaxy formation down to $z=2$, together with analytic models of black hole accretion, to investigate the growth of massive black holes at the centers of galaxies without making any prior assumptions about the effects of AGN feedback. To this end, we have compared predictions from the spherical Bondi model and an accretion model driven by gravitational torques (Hopkins \& Quataert 2011).
We find that the Bondi model presents significant challenges due to the strong dependence of the accretion rates on black hole mass. Black hole growth is significantly suppressed for low-mass black holes and becomes extremely rapid for sufficiently massive black holes. This has two main implications for simulations of galaxy formation: (1) the initial black hole mass (together with any boost factor or normalization constant) has to be sufficiently massive to allow for early black hole growth and (2) feedback energy and/or momentum need to be injected into the surrounding gas in order to regulate black hole growth at later times. Because these implications follow from the $\propto M_{\mathrm{BH}}^{2}$ dependence, they should apply to any (reasonable) modification of the original Bondi-Hoyle-Lyttleton parameterization. Remarkably, suitable choices of model parameters for different implementations have been successful in reproducing a broad number of observations, including the black hole-galaxy correlations (e.g., Di Matteo et al. 2005; Robertson et al. 2006; Hopkins et al. 2007; Booth \& Schaye 2009; Johansson et al. 2009).

In the gravitational torque model, gas inflows are driven by global gravitational instabilities in the disk and, therefore, do not depend strongly on the black hole mass (Hopkins \& Quataert 2011). The resulting accretion rates imply that (1) early black hole growth could in principle proceed at super-critical rates, since the inflow rates can be well above the Eddington limit even for small initial black holes, and (2) energy (and/or momentum) from the accretion process does not need to couple to galaxyscale gas in order to regulate black hole growth. Consistency with the black hole-galaxy correlations simply requires the assumption that only a small (constant) fraction of the mass inflow is retained in the accretion flow, with the rest lost to winds and outflows. We have shown that this result is insensitive to variations in the initial black hole mass, stellar feedback, or other implementation details, and the required mass retention rate $\left(\epsilon_{\mathrm{m}} \approx 5 \%\right)$ is roughly consistent with observational (e.g., King et al. 2013) and theoretical expectations (e.g., Yuan et al. 2012, and references therein). However, the exact value of the normalization factor $\epsilon_{\mathrm{m}}$ that we infer is subject to uncertainties such as variations in the nuclear star formation law or the exact form and normalization of the $M_{\mathrm{BH}}-M_{\text {bulge }}$ relation (see Graham 2012).

Mass outflows are invoked in the torque-limited model in order to control what fraction $\left(\epsilon_{\mathrm{m}}\right)$ of the accretion disk feeding rate is finally accreted by the central black hole (a linear effect), but not to regulate the amount of gas feeding the accretion disk from galactic scales. In this scenario, there is no coupling between inflows and outflows; therefore, black hole feedback cannot shut down accretion. "Feedback self-regulation" does not occur in the strict sense of the term, since the energy output by accretion onto the black hole has no direct impact on its own fuel supply. This greatly differs from the non-linear feedback loop required by the Bondi model. Large-scale AGN feedback may have a significant impact on the host galaxy, but it is not required for regulating black hole growth, which may instead be limited by the efficiency of gravitational torques in removing angular momentum from the gas together with competition with star formation.

In addition, torque-limited growth yields a less direct correspondence between major merger events and enhanced AGN activity, as suggested by recent observations of $z \sim 2$ active galaxies (Kocevski et al. 2012; Mullaney et al. 2012b; Rosario et al. 2012; Schawinski et al. 2012). Instead, black hole and galaxy growth are governed by cosmological infall 
and transport of angular momentum in the galactic disk, giving rise to a time-averaged connection between AGN activity and SFR on cosmological timescales. Our findings are consistent with recent observational evidence for a constant ratio of black hole accretion to star formation, similar to the ratio of black hole mass to stellar mass as inferred from the local $M_{\mathrm{BH}}-M_{\text {bulge }}$ relation, and independent of redshift since $z \sim 2$ (Rafferty et al. 2011; Mullaney et al. 2012a).

The agreement of feedback-regulated accretion models in hydrodynamic simulations to the observed black hole-galaxy correlations has been interpreted as (indirect) observational evidence of both AGN feedback acting at galactic scales and the self-regulated growth of black holes. We note, however, that the black hole-galaxy correlations are indeed a primary constraint for the strength of such feedback models (see Debuhr et al. 2011 for a self-regulated model that accounts for angular momentum transport). Given that AGN feedback can have profound consequences on the evolution of the host galaxy (e.g., Di Matteo et al. 2005) and that the scaling relations are not necessarily a consequence of feedback, it would be desirable to constrain AGN feedback models by other means, ideally through direct observations of mass outflow rates (e.g., Feruglio et al. 2010) and/or more physically motivated models (e.g., Ciotti \& Ostriker 2007; Novak et al. 2011, 2012). While the Bondi model, appropriately tuned, does provide a good match to the black hole-galaxy correlations, our work demonstrates that the torque-limited model provides a viable alternative that does not require self-regulation.

Our findings should apply so long as the assumptions implied by the gravitational torque model are met. In particular, it is assumed that (1) there is a significant stellar component that drives the gas into shocks that dissipate energy and angular momentum and (2) the amplitudes of non-axisymmetric modes are large enough to produce such shocks even at $\sim 10$ pc scales (Hopkins \& Quataert 2011). These scales are, of course, not resolved in our simulations; galaxy centers become quickly stellar dominated, but the formation time of the first star particles is poorly constrained. Other processes such as scattering of dense gas clouds or the transport of angular momentum by supernova and/or gravitational instability-driven turbulence may be required in the very high gas-reach domain (e.g., Escala 2006; Bournaud et al. 2011; Hobbs et al. 2011; Gaspari et al. 2013). Finally, full cosmological simulations with adequate resolution down to $z=0$ are required in order to evaluate the implications of torque-limited growth on a statistically significant sample of galaxies, making connection with the wealth of data available in the local and low-redshift universe over a range of galaxy masses spanning several orders of magnitude.

We thank K. Finlator, P. Hopkins, B. Oppenheimer, D. Psaltis, and B. Robertson for stimulating conversations, and the anonymous referee for constructive comments that helped improve the paper. D.A.-A. thanks B. Robertson for very useful assistance with data visualization. F.O. gratefully acknowledges support from the Radcliffe Institute for Advanced Study at Harvard University. The simulations were run on the University of Arizona's 512-processor SGI Altix system and the TACC Sun Constellation Cluster (Ranger) at the University of Texas, Austin. This work used the Extreme Science and Engineering Discovery Environment (XSEDE), which is supported by National Science Foundation grant number OCI-1053575. This work was supported by the National
Science Foundation under grant numbers AST-0907998 and AST-1108753. Computing resources were obtained through grant number DMS-0619881 from the National Science Foundation.

\section{REFERENCES}

Alexander, D. M., \& Hickox, R. C. 2012, NewAR, 56, 93

Aller, M. C., \& Richstone, D. O. 2007, ApJ, 665, 120

Anglés-Alcázar, D., Davé, R., Özel, F., \& Oppenheimer, B. D. 2013, arXiv:1303.6959

Balbus, S. A., \& Hawley, J. F. 1998, RvMP, 70, 1

Barnes, J. E., \& Hernquist, L. 1992, ARA\&A, 30, 705

Begelman, M. C., \& Nath, B. B. 2005, MNRAS, 361, 1387

Böhm, A., Wisotzki, L., Bell, E. F., et al. 2013, A\&A, 549, A46

Bondi, H. 1952, MNRAS, 112, 195

Bondi, H., \& Hoyle, F. 1944, MNRAS, 104, 273

Booth, C. M., \& Schaye, J. 2009, MNRAS, 398, 53

Bournaud, F., Dekel, A., Teyssier, R., et al. 2011, ApJL, 741, L33

Boyle, B. J., \& Terlevich, R. J. 1998, MNRAS, 293, L49

Cen, R. 2012, ApJ, 755, 28

Choi, E., Ostriker, J. P., Naab, T., \& Johansson, P. H. 2012, ApJ, 754, 125

Ciotti, L., \& Ostriker, J. P. 2007, ApJ, 665, 1038

Cisternas, M., Jahnke, K., Bongiorno, A., et al. 2011a, ApJL, 741, L11

Cisternas, M., Jahnke, K., Inskip, K. J., et al. 2011b, ApJ, 726, 57

Croton, D. J., Springel, V., White, S. D. M., et al. 2006, MNRAS, 365, 11

Davé, R., Finlator, K., \& Oppenheimer, B. D. 2006, MNRAS, 370, 273

Davé, R., Oppenheimer, B. D., \& Finlator, K. 2011, MNRAS, 415, 11

Debuhr, J., Quataert, E., \& Ma, C.-P. 2011, MNRAS, 412, 1341

Debuhr, J., Quataert, E., \& Ma, C.-P. 2012, MNRAS, 420, 2221

Decarli, R., Falomo, R., Treves, A., et al. 2010, MNRAS, 402, 2453

De Rosa, G., Decarli, R., Walter, F., et al. 2011, ApJ, 739, 56

Di Matteo, T., Colberg, J., Springel, V., Hernquist, L., \& Sijacki, D. 2008, ApJ, 676,33

Di Matteo, T., Quataert, E., Allen, S. W., Narayan, R., \& Fabian, A. C. 2000, MNRAS, 311, 507

Di Matteo, T., Springel, V., \& Hernquist, L. 2005, Natur, 433, 604

Dubois, Y., Devriendt, J., Slyz, A., \& Teyssier, R. 2012, MNRAS, 420, 2662

Escala, A. 2006, ApJL, 648, L13

Escala, A. 2007, ApJ, 671, 1264

Fabian, A. C. 1999, MNRAS, 308, L39

Fabian, A. C. 2012, ARA\&A, 50, 455

Fan, X., Strauss, M. A., Schneider, D. P., et al. 2003, AJ, 125, 1649

Ferrarese, L. 2002, ApJ, 578, 90

Ferrarese, L., \& Ford, H. 2005, SSRv, 116, 523

Ferrarese, L., \& Merritt, D. 2000, ApJL, 539, L9

Feruglio, C., Maiolino, R., Piconcelli, E., et al. 2010, A\&A, 518, L155

Finlator, K., \& Davé, R. 2008, MNRAS, 385, 2181

Finlator, K., Davé, R., \& Oppenheimer, B. D. 2007, MNRAS, 376, 1861

Förster Schreiber, N. M., Genzel, R., Bouché, N., et al. 2009, ApJ, 706, 1364

Gabor, J. M., Davé, R., Oppenheimer, B. D., \& Finlator, K. 2011, MNRAS, 417, 2676

Gabor, J. M., Impey, C. D., Jahnke, K., et al. 2009, ApJ, 691, 705

Gaspari, M., Ruszkowski, M., \& Oh, S. P. 2013, arXiv:1301.3130

Graham, A. W. 2012, ApJ, 746, 113

Graham, A. W., Erwin, P., Caon, N., \& Trujillo, I. 2001, ApJL, 563, L11

Greene, J. E., Peng, C. Y., \& Ludwig, R. R. 2010, ApJ, 709, 937

Gültekin, K., Richstone, D. O., Gebhardt, K., et al. 2009, ApJ, 698, 198

Haardt, F., \& Madau, P. 2001, in Clusters of Galaxies and the High Redshift Universe Observed in X-Rays, ed. D. M. Neumann \& J. T. T. Van (Paris: Editions Frontiéres), 64

Hambrick, D. C., Ostriker, J. P., Naab, T., \& Johansson, P. H. 2011, ApJ, 738,16

Häring, N., \& Rix, H. W. 2004, ApJ, 604, 89

Heckman, T. M., Kauffmann, G., Brinchmann, J., et al. 2004, ApJ, 613, 109

Hernquist, L. 1989, Natur, 340, 687

Hobbs, A., Nayakshin, S., Power, C., \& King, A. 2011, MNRAS, 413, 2633

Hobbs, A., Power, C., Nayakshin, S., \& King, A. R. 2012, MNRAS, 421, 3443

Hopkins, P. F., Hernquist, L., Cox, T. J., Robertson, B., \& Krause, E. 2007, ApJ, 669,45

Hopkins, P. F., Hernquist, L., Cox, T. J., et al. 2006, ApJS, 163, 1

Hopkins, P. F., \& Quataert, E. 2010, MNRAS, 407, 1529

Hopkins, P. F., \& Quataert, E. 2011, MNRAS, 415, 1027

Hoyle, F., \& Lyttleton, R. A. 1939, PCPS, 34, 405

Jahnke, K., Bongiorno, A., Brusa, M., et al. 2009, ApJL, 706, L215

Jahnke, K., \& Macciò, A. V. 2011, ApJ, 734, 92 
Jogee, S. 2006, in AGN Physics on All Scales, ed. D. Alloin, R. Johnson, \& P. Lira (Lecture Notes in Physics, Vol. 93; Berlin: Springer), 143

Johansson, P. H., Naab, T., \& Burkert, A. 2009, ApJ, 690, 802

Katz, N., Weinberg, D. H., \& Hernquist, L. 1996, ApJS, 105, 19

Kauffmann, G., Heckman, T. M., Tremonti, C., et al. 2003, MNRAS, 346, 1055

Kennicutt, R. C. 1998, ApJ, 498, 541

Keres, D., Katz, N., Weinberg, D. H., \& Davé, R. 2005, MNRAS, 363, 2

Kim, J.-H., Wise, J. H., Alvarez, M. A., \& Abel, T. 2011, ApJ, 738, 54

King, A. 2003, ApJL, 596, L27

King, A. L., Miller, J. M., Raymond, J., et al. 2013, ApJ, 762, 103

Kitayama, T., \& Suto, Y. 1996, ApJ, 469, 480

Kocevski, D. D., Faber, S. M., Mozena, M., et al. 2012, ApJ, 744, 148

Komatsu, E., Dunkley, J., Nolta, M. R., et al. 2009, ApJS, 180, 330

Lauer, T. R., Tremaine, S., Richstone, D., \& Faber, S. M. 2007, ApJ, 670, 249

Madau, P., Ferguson, H. C., Dickinson, M. E., et al. 1996, MNRAS, 283, 1388

Madau, P., \& Rees, M. J. 2001, ApJL, 551, L27

Magorrian, J., Tremaine, S., Richstone, D., et al. 1998, AJ, 115, 2285

Marconi, A., \& Hunt, L. K. 2003, ApJL, 589, L21

McConnell, N. J., Ma, C.-P., Gebhardt, K., et al. 2011, Natur, 480, 215

McKee, C. F., \& Ostriker, J. P. 1977, ApJ, 218, 148

McQuillin, R. C., \& McLaughlin, D. E. 2012, MNRAS, 423, 2162

Merloni, A., Bongiorno, A., Bolzonella, M., et al. 2010, ApJ, 708, 137

Mo, H. J., Mau, S., \& White, S. D. M. 1998, MNRAS, 295, 319

Mortlock, D. J., Warren, S. J., Venemans, B. P., et al. 2011, Natur, 474, 616

Mullaney, J. R., Daddi, E., Béthermin, M., et al. 2012a, ApJL, 753, L30

Mullaney, J. R., Pannella, M., Daddi, E., et al. 2012b, MNRAS, 419, 95

Murali, C., Katz, N., Hernquist, L., Weinberg, D. H., \& Davé, R. 2002, ApJ, 571,1

Murray, N., Quataert, E., \& Thompson, T. A. 2005, ApJ, 618, 569

Navarro, J. F., \& White, S. D. M. 1994, MNRAS, 267, 401

Novak, G. S., Ostriker, J. P., \& Ciotti, L. 2011, ApJ, 737, 26

Novak, G. S., Ostriker, J. P., \& Ciotti, L. 2012, MNRAS, 427, 2734

Ohsuga, K., Mori, M., Nakamoto, T., \& Mineshige, S. 2005, ApJ, 628, 368

Oppenheimer, B. D., \& Davé, R. 2006, MNRAS, 373, 1265
Oppenheimer, B. D., \& Davé, R. 2008, MNRAS, 387, 577

Pelupessy, F. I., Di Matteo, T., \& Ciardi, B. 2007, ApJ, 665, 107

Peng, C. Y. 2007, ApJ, 671, 1098

Power, C., Nayakshin, S., \& King, A. 2011, MNRAS, 412, 269

Proga, D., Stone, J. M., \& Kallman, T. R. 2000, ApJ, 543, 686

Rafferty, D. A., Brandt, W. N., Alexander, D. M., et al. 2011, ApJ, 742, 3

Robertson, B., Hernquist, L., Cox, T. J., et al. 2006, ApJ, 641, 90

Rosario, D. J., Santini, P., Lutz, D., et al. 2012, A\&A, 545, A45

Sales, L. V., Navarro, J. F., Schaye, J., et al. 2010, MNRAS, 409, 1541

Schawinski, K., Simmons, B. D., Urry, C. M., Treister, E., \& Glikman, E. 2012, MNRAS, 425, L61

Shakura, N. I., \& Sunyaev, R. A. 1973, A\&A, 24, 337

Shapiro, K. L., Genzel, R., Quataert, E., et al. 2009, ApJ, 701, 955

Shen, J. J., Vanden Berk, D. E., Schneider, D. P., \& Hall, P. B. 2008, AJ, 135,928

Shlosman, I., Frank, J., \& Begelman, M. C. 1989, Natur, 338, 45

Sijacki, D., Springel, V., Di Matteo, T., \& Hernquist, L. 2007, MNRAS, 380,877

Silk, J., \& Rees, M. J. 1998, A\&A, 331, L1

Soltan, A. 1982, MNRAS, 200, 115

Somerville, R. S., Hopkins, P. F., Cox, T. J., Robertson, B. E., \& Hernquist, L. 2008, MNRAS, 391, 481

Springel, V. 2005, MNRAS, 364, 1105

Springel, V., Di Matteo, T., \& Hernquist, L. 2005, MNRAS, 61, 776

Springel, V., \& Hernquist, L. 2002, MNRAS, 333, 649

Springel, V., \& Hernquist, L. 2003, MNRAS, 339, 289

Sutherland, R. S., \& Dopita, M. A. 1993, ApJS, 88, 253

Teyssier, R., Moore, B., Martizzi, D., Dubois, Y., \& Mayer, L. 2011, MNRAS, 414, 195

Thompson, T. A., Quataert, E., \& Murray, N. 2005, ApJ, 630, 167

Trakhtenbrot, B., Netzer, H., Lira, P., \& Shemmer, O. 2011, ApJ, 730, 7

Tremaine, S., Gebhardt, K., Bender, R., et al. 2002, ApJ, 574, 740

Volonteri, M. 2010, A\&AR, 18, 279

Woo, J., Treu, T., Malkan, M. A., \& Blandford, R. D. 2008, ApJ, 681, 925

Yuan, F., Bu, D., \& Wu, M. 2012, ApJ, 761, 130

Zheng, X. Z., Bell, E. F., Somerville, R. S., et al. 2009, ApJ, 707, 1566 